\title{
VLBI observations of optically-bright extragalactic radio sources for the alignment of the radio frame with the future Gaia frame
}

\section{Imaging candidate sources ${ }^{\star}$}

\author{
G. Bourda ${ }^{1,2}$, A. Collioud ${ }^{1,2}$, P. Charlot ${ }^{1,2}$, R. Porcas ${ }^{3}$, and S. Garrington ${ }^{4}$ \\ 1 Université de Bordeaux, Observatoire Aquitain des Sciences de l'Univers, 2 rue de l'Observatoire, BP 89, 33271 Floirac Cedex, \\ France \\ e-mail: bourda@obs.u-bordeaux1.fr \\ 2 CNRS, UMR 5804, Laboratoire d'Astrophysique de Bordeaux, 2 rue de l'Observatoire, BP 89, 33271 Floirac Cedex, France \\ 3 Max-Planck-Institut für Radioastronomie, Auf dem Hügel 69, 53121 Bonn, Germany \\ 4 University of Manchester, Jodrell Bank Observatory, Macclesfield, Cheshire SK11 9DL, UK
}

Received 11 February 2010 / Accepted 25 October 2010

\begin{abstract}
Context. The European space astrometry mission Gaia, to be launched by 2012, will construct a dense optical QSO-based celestial reference frame which will need to be linked to the International Celestial Reference Frame (ICRF; the IAU fundamental frame), with the highest accuracy. However, it has been found that only $10 \%$ of the ICRF sources (70 sources) are suitable to establish this link. The remaining sources are not useful either because they are not bright enough at optical wavelengths or because they have significant extended radio emission which precludes reaching the highest astrometric accuracy.

Aims. In order to improve the accuracy of this alignment, we have developed a program of VLBI observations based on three steps to detect, image and measure astrometric positions of weak extragalactic radio sources, with bright optical counterparts, from a sample of 447 candidate sources.

Methods. The experiments devoted to VLBI detection, carried out with the European VLBI Network (EVN) in June and October 2007, were very successful, with 398 sources detected at both $S$ - and $X$-bands. From these, 105 sources were observed in March 2008 with a global VLBI array (EVN and VLBA; Very Long Baseline Array) for imaging their VLBI structures.

Results. All sources were successfully imaged in both bands and about 50\% (47 sources) were found to be point-like on VLBI scales. These images are available at http://www.obs.u-bordeaux1.fr/BVID/GCQ30/. VLBI positions of these sources will be measured accurately in future astrometric experiments.
\end{abstract}

Key words. reference systems - quasars: general - astrometry - catalogs - methods: observational - techniques: interferometric

\section{Introduction}

Since 1 January 2010 the fundamental celestial reference frame recognized by the IAU (International Astronomical Union) has been the ICRF2 (the successor of the ICRF, the International Celestial Reference Frame; Ma et al. 1998; Fey et al. 2004). The ICRF2 (described in IERS Technical Note 35) includes VLBI (Very Long Baseline Interferometry) coordinates of 3414 extragalactic radio sources (i.e. quasars, active galaxies or BL Lac objects), with a floor in position accuracy of about 60 microarcseconds ( $\mu$ as). The European space astrometry mission Gaia, to be launched by 2012, will survey all stars and QSOs (Quasi Stellar Objects) brighter than apparent optical magnitude 20 (Perryman et al. 2001), with an unprecedented accuracy for optical wavelengths (a few tens of $\mu$ as at magnitude 15-18; Lindegren et al. 2008). Gaia will permit the determination of the extragalactic celestial reference frame directly in the optical domain. By 2015-2020, when the Gaia catalog is available, both the radio and optical celestial reference frames will coexist, with similar accuracies. At this stage, aligning them will be crucial to ensure

* Full Table 3 is only available in electronic form at the CDS via anonymous ftp to cdsarc.u-strasbg.fr $(130.79 .128 .5)$ or via http://cdsweb.u-strasbg.fr/viz-bin/qcat?]/A+A/526/A102 consistency between the measured radio and optical positions. Such an alignment, to be achieved with the highest accuracy, will require optically-bright extragalactic radio sources of good astrometric quality, as pointed out by Bourda et al. (2008, 2010).

The need to find new VLBI sources suitable for this alignment, which has already been highlighted (Bourda et al. 2008), requires going to sources with weaker flux densities than those observed so far with VLBI. To this end, a VLBI observational program in three successive steps has been developed (Bourda et al. 2010). This multi-step VLBI project is based on the observation of a sample of 447 optically-bright, weak extragalactic radio sources selected from the NVSS catalog (NRAO VLA Sky Survey; Condon et al. 1998), with declinations above $-10^{\circ}$, and flux densities at $1.4 \mathrm{GHz}$ greater than $20 \mathrm{mJy}$. This program consists of the following successive stages:

1. detection experiments with the European VLBI Network $(\mathrm{EVN})$, in order to identify those of the 447 candidate sources that are "visible" with VLBI;

2. an imaging survey of the targets detected in step 1 with a global VLBI array (EVN and VLBA; Very Long Baseline Array), in order to identify the most point-like sources, which have the highest astrometric quality; 
3 . an astrometric survey of the most compact sources identified in step 2, in order to determine highly accurate VLBI positions.

VLBI detection was the purpose of two 48-h $S / X$-band experiments $(2.3 / 8.4 \mathrm{GHz})$ conducted with the EVN in June and October 2007 (experiments designated EC025A and EC025B, respectively). In total 398 sources were detected at both $S$ - and $X$-bands as reported in Bourda et al. (2010).

In this paper we report on VLBI imaging of 105 of these sources, selected from those detected during experiment EC025A. More specifically, we focused on the strongest sources (i.e. those with a mean correlated flux density at $X$-band larger than $33 \mathrm{mJy}$ ). This represents about one quarter of the sources to be observed in step 2 of our observational program. First, the observations and data reduction procedures for VLBI imaging are described. Then the resulting VLBI maps are presented and discussed. In this investigation the continuous structure index (SI) is used to identify the sources that have the most compact VLBI brightness distributions. The end result is a set of 47 sources suitable for the VLBI-Gaia frame alignment, to be observed in step 3 of our project.

\section{Observations and data reduction}

The observations were performed during a 48-h experiment (designated GC030) on 7-9 March 2008, with a global VLBI array recording at $512 \mathrm{Mb} / \mathrm{s}$ - the maximum recording rate available with the VLBA - in a dual-frequency S/X mode with the setup as shown in Table 1. The network comprised 5 telescopes of the EVN (Effelsberg, Medicina, Noto, Onsala-25 m and the South-African antenna at Hartebeesthoek), the DSN 70$\mathrm{m}$ Robledo telescope for part of the time, and 9 antennas of the VLBA (Napier et al. 1994); the VLBA Fort Davis antenna could not observe during this experiment. The participation of the $100 \mathrm{~m}$ Effelsberg telescope was essential in reaching the necessary baseline sensitivity, since our targets are typically 27 times weaker than ICRF sources on average (Bourda et al. 2010). The Hartebeesthoek antenna was included in order to improve the North-South $(u, v)$ coverage, since approximately half of our targets are below $30^{\circ}$ declination. The maximum angular resolution, obtained with the baseline Mauna Kea-Hartebeesthoek, is about 0.7 mas at $X$-band and 2.6 mas at $S$-band.

On average a total of three to four 5-min-long scans were scheduled on each of the 105 target sources. This small number of scans per source is sufficient because of the large number of elements in the network. Considering a source as detected if $\mathrm{SNR} \geq 7$, the theoretical minimum detectable flux density on given baselines during GC030 can be estimated (see Table 2). Accordingly, on the basis of the values obtained for all the baselines to Effelsberg, all sources in the observed sample should be detected.

In addition we observed a sample of 10 ICRF sources, well-distributed on the sky, for use as calibrators: $0059+581$, $0133+476,0229+131,0552+398,0805+410,1044+719$, $1219+044,1739+522,1749+096$, and $2113+293$. In all about $80 \%$ of the allocated time was spent on-source, while the rest was used for slewing.

The GC030 correlation was done with the VLBA correlator at the Array Operations Center in Socorro (New Mexico). The correlated data were then calibrated using the Astronomical Image Processing System (AIPS ${ }^{1}$; Greisen 1988) in a standard

\footnotetext{
$\overline{1}$ http://www .aips.nrao . edu/cook.html
}

Table 1. The sub-bands used in global experiment GC030: for each frequency there are 8 sub-bands of $8 \mathrm{MHz}$ bandwidth.

\begin{tabular}{lcc}
\hline \hline Sub-bands & $\begin{array}{c}X \text {-band central } \\
\text { frequency (MHz) }\end{array}$ & $\begin{array}{c}S \text {-band central } \\
\text { frequency (MHz) }\end{array}$ \\
\hline 1 & 8380.99 & 2230.99 \\
2 & 8388.99 & 2238.99 \\
3 & 8396.99 & 2246.99 \\
4 & 8404.99 & 2254.99 \\
5 & 8412.99 & 2262.99 \\
6 & 8420.99 & 2270.99 \\
7 & 8428.99 & 2278.99 \\
8 & 8436.99 & 2286.99 \\
\hline
\end{tabular}

way. An initial amplitude calibration for each sub-band was made using system temperature measurements taken during the observations, combined with gain curves supplied for each telescope. Then, in order to fringe-fit the data from these relatively weak sources, it was necessary to first combine the sub-bands; this was achieved by performing a "manual" phase calibration, i.e. determining phase offsets in each sub-band by fringe-fitting a short, calibrator scan, and then applying them to all the data. Fringe-fitting then produced corrections for residual delays and delay rates. Calibrators were used in order to estimate residual global amplitude correction factors for each telescope, band ( $S$ and $X$ ) and sub-band. These corrections, typically less than $10 \%$ on average, were applied to the calibrated data. Finally, the data were exported as FITS files.

In a second step the FITS files were imported to the Caltech DIFMAP ${ }^{2}$ software-package (Shepherd 1997). Visibility data for both frequency bands were self-calibrated, Fourier inverted, and CLEANed using the hybrid-mapping technique (Pearson \& Readhead 1984), employing DIFMAP in an automatic mode. A point-source model was used as a starting model for this iterative procedure in all cases. Uniform weighting and, after several iterations, natural weighting, were successively applied to derive the final images.

\section{Results}

From this analysis, VLBI maps at $X$ - and $S$-bands were produced for each of the 105 sources observed during GC030 and listed in Table $3^{3}$. Figures 1 and 2 show examples of these maps for the first 12 sources in the list. The maps for all 105 target sources are given in appendix and can be accessed on-line ${ }^{4}$.

The parameters of these naturally-weighted images (i.e. beamsize, peak brightness, rms noise, and contour levels) are given in Table 3. The typical restoring beam has a size of about $1.2 \times 0.5$ mas at $X$-band and $4.2 \times 2.0$ mas at $S$-band. In these images the first contour level is typically $1 \%$ of the peak brightness, with successive levels increasing by a factor of 2. Figure 3 shows the peak brightness plotted against the rms noise for each image (i.e. for each source at both bands). The dynamic range is usually $\sim 1: 100$. The typical image rms

\footnotetext{
2 http://www . astro.caltech.edu/ tjp/citvlb/index.html 3 Complete Table 3 is available in electronic form at the CDS.

4 The VLBI images at $X$ - and $S$-bands for the 105 target sources are available from the Bordeaux VLBI Image Database (BVID; see http: //WWw . obs. u-bordeaux 1. fr/BVID/GCQ30/ for more details).
} 
G. Bourda et al.: VLBI observations of optically-bright extragalactic radio sources. II.
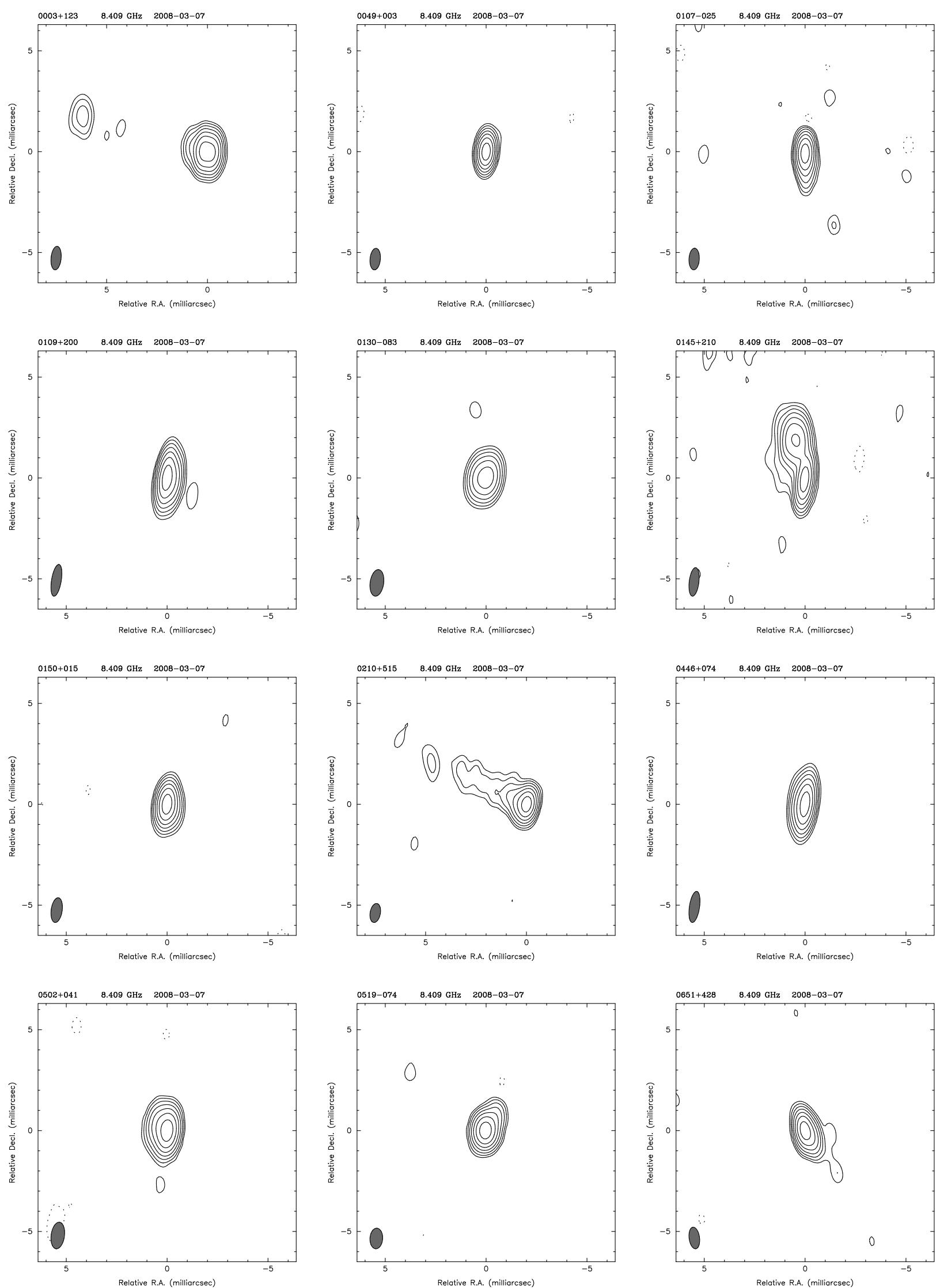

Fig. 1. Examples of VLBI maps (at $X$-band) for the first 12 sources from GC030 listed in Table $3(0003+123,0049+003,0107-025,0109+200$, $0130-083,0145+210,0150+015,0210+515,0446+074,0502+041,0519-074$ and $0651+428)$. Image parameters are listed in Table 3. The first contour level is typically $1 \%$ of the peak brightness, with successive contours increasing by a factor of 2 . The FWHM Gaussian restoring beam applied to the images is shown as an ellipse in the lower left of each panel. 

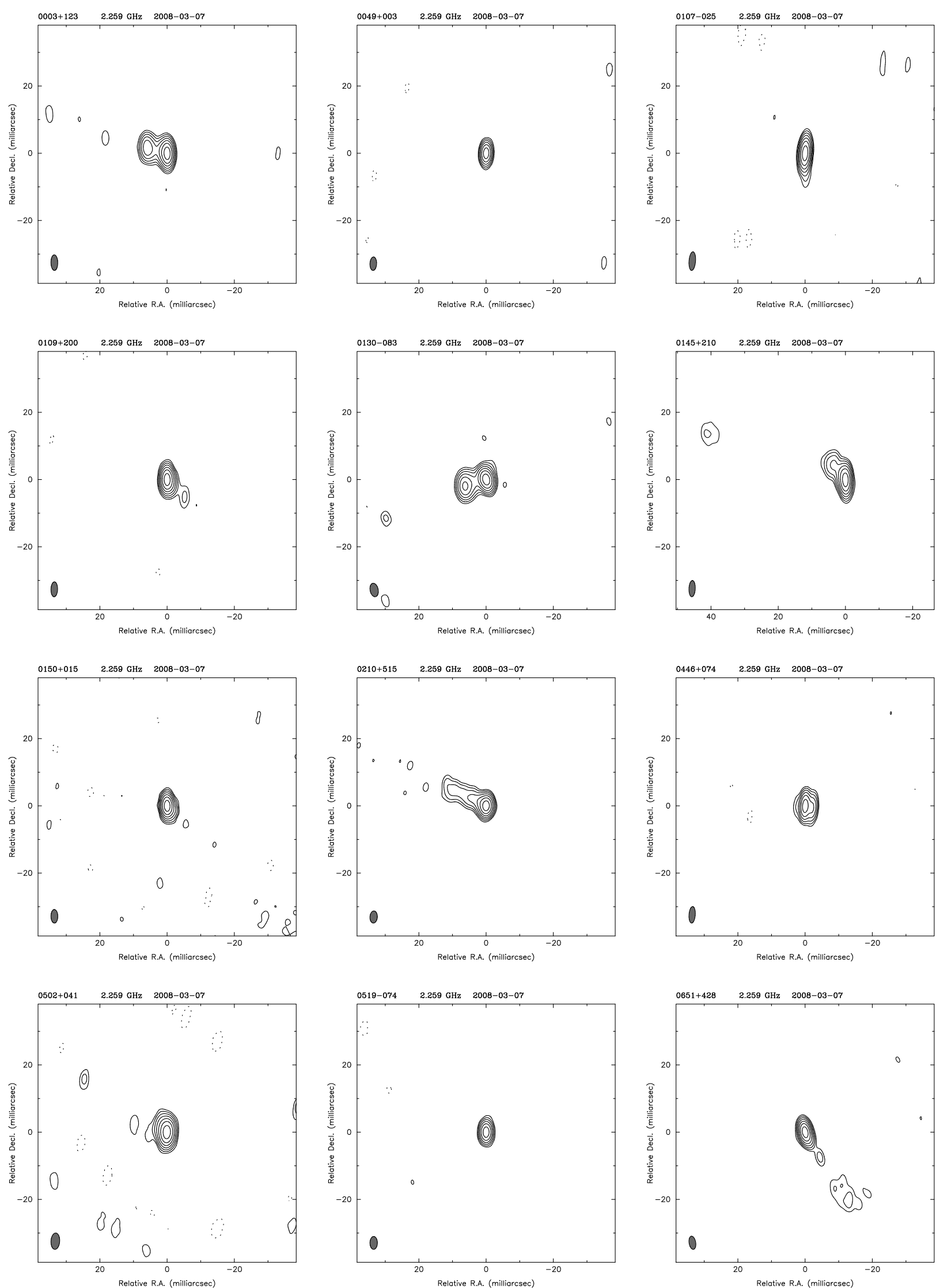

Fig. 2. Examples of VLBI maps (at $S$-band) for the first 12 sources from GC030 listed in Table $3(0003+123,0049+003,0107-025$, 0109+200, $0130-083,0145+210,0150+015,0210+515,0446+074,0502+041,0519-074$ and $0651+428)$. Image parameters are listed in Table 3. The first contour level is typically $1 \%$ of the peak brightness, with successive contours increasing by a factor of 2 . The FWHM Gaussian restoring beam applied to the images is shown as an ellipse in the lower left of each panel. 
G. Bourda et al.: VLBI observations of optically-bright extragalactic radio sources. II.

Table 2. Theoretical estimate of the minimum detectable flux density with the baselines given below, assuming $\mathrm{S} / \mathrm{X}$ observations recording at $512 \mathrm{Mb} / \mathrm{s}$ and 5-minute-long scans.

\begin{tabular}{ccccccccc}
\hline \hline & & \multicolumn{7}{c}{ Minimum detectable flux density (mJy) } \\
& Eb-VLBA & Eb-Mc & Eb-Nt & Eb-On & Eb-Ro & Eb-Hh & VLBA-VLBA & VLBA-Mc \\
\hline$X$-band & 4 & 4 & 6 & 7 & 1 & 7 & 16 & 16 \\
$S$-band & 16 & 18 & 25 & 29 & 4 & 17 & 16 & 18 \\
\hline
\end{tabular}

Notes. $A$ detection corresponds to $\mathrm{SNR} \geq 7$. The names of the observing stations are: $\mathrm{Eb}=$ Effelsberg, $\mathrm{Mc}=\mathrm{Medicina}, \mathrm{Nt}=\mathrm{Noto}, \mathrm{On}=\mathrm{Onsala}$, Ro $=$ Robledo, $\mathrm{Hh}=$ Hartebeestoek, and VLBA $=$ Very Long Baseline Array .

Table 3. Sample of 12 sources extracted from the list of 105 optically-bright, weak extragalactic radio sources, observed during GC030. The complete list is available at the CDS.

\begin{tabular}{|c|c|c|c|c|c|c|c|c|c|c|c|}
\hline \multirow[b]{2}{*}{ IERS name } & \multirow[b]{2}{*}{ Band } & \multirow[b]{2}{*}{$\begin{array}{r}\text { Flux density } \\
\text { mJy }\end{array}$} & \multirow[b]{2}{*}{$\alpha_{\mathrm{sx}}$} & \multirow[b]{2}{*}{ SI } & \multicolumn{3}{|c|}{ Beam } & \multirow[b]{2}{*}{$\begin{array}{r}\text { Peak } \\
\text { mJy/beam }\end{array}$} & \multirow[b]{2}{*}{$\begin{array}{r}\mathrm{rms} \\
\mathrm{mJy} / \text { beam }\end{array}$} & \multicolumn{2}{|c|}{ First contour level } \\
\hline & & & & & $\begin{array}{c}a \\
\text { mas }\end{array}$ & $\begin{array}{c}b \\
\text { mas }\end{array}$ & $\begin{array}{c}\phi \\
\text { deg }\end{array}$ & & & $\begin{array}{c}\text { First } \\
\%\end{array}$ & $\begin{array}{l}\text { ntour level } \\
\text { mJy/beam }\end{array}$ \\
\hline \multirow[t]{2}{*}{$0003+123$} & $X$ & 127.9 & 0.1 & 3.9 & 1.17 & 0.50 & -5.6 & 22.4 & 0.07 & $1 \%$ & 0.22 \\
\hline & $S$ & 115.4 & & 2.6 & 4.64 & 2.03 & 0.9 & 52.2 & 0.12 & $1 \%$ & 0.52 \\
\hline \multirow[t]{2}{*}{$0049+003$} & $X$ & 48.9 & 0.4 & 0.0 & 1.07 & 0.51 & -5.4 & 49.3 & 0.06 & $1 \%$ & 0.49 \\
\hline & $S$ & 28.3 & & 2.5 & 4.01 & 1.98 & -2.5 & 34.1 & 0.15 & $2 \%$ & 0.68 \\
\hline \multirow[t]{2}{*}{$0107-025$} & $X$ & 46.0 & -0.2 & 3.8 & 1.07 & 0.51 & -2.9 & 13.0 & 0.13 & $1 \%$ & 0.13 \\
\hline & $S$ & 56.3 & & 1.9 & 5.57 & 1.94 & -4.5 & 51.4 & 0.15 & $1 \%$ & 0.51 \\
\hline \multirow[t]{2}{*}{$0109+200$} & $X$ & 120.3 & 0.3 & 2.5 & 1.59 & 0.48 & -9.4 & 65.0 & 0.07 & $1 \%$ & 0.64 \\
\hline & $S$ & 77.8 & & 1.1 & 4.43 & 1.97 & -1.6 & 53.9 & 0.11 & $1 \%$ & 0.54 \\
\hline \multirow[t]{2}{*}{$0130-083$} & $X$ & 78.3 & -0.5 & 4.1 & 1.33 & 0.67 & -8.1 & 19.5 & 0.11 & $2 \%$ & 0.39 \\
\hline & $S$ & 140.3 & & 2.7 & 4.07 & 2.41 & 11.6 & 54.7 & 0.14 & $1 \%$ & 0.55 \\
\hline \multirow[t]{2}{*}{$0145+210$} & $X$ & 126.4 & -0.6 & 4.4 & 1.43 & 0.48 & -6.8 & 22.5 & 0.13 & $1 \%$ & 0.23 \\
\hline & $S$ & 267.9 & & 1.6 & 4.83 & 1.87 & -2.4 & 131.4 & 0.14 & $1 \%$ & 1.31 \\
\hline \multirow[t]{2}{*}{$0150+015$} & $X$ & 44.5 & 0.1 & 3.0 & 1.24 & 0.55 & -8.1 & 31.7 & 0.10 & $1 \%$ & 0.32 \\
\hline & $S$ & 36.9 & & 2.7 & 4.03 & 2.04 & 0.6 & 34.8 & 0.13 & $1 \%$ & 0.35 \\
\hline \multirow[t]{2}{*}{$0210+515$} & $X$ & 70.7 & -0.4 & 2.6 & 0.95 & 0.51 & -10.0 & 31.5 & 0.08 & $1 \%$ & 0.32 \\
\hline & $S$ & 114.1 & & 1.1 & 3.61 & 2.19 & -3.8 & 55.4 & 0.11 & $1 \%$ & 0.55 \\
\hline \multirow[t]{2}{*}{$0446+074$} & $X$ & 89.3 & 0.5 & 1.8 & 1.56 & 0.50 & -8.5 & 59.4 & 0.06 & $1 \%$ & 0.59 \\
\hline & $S$ & 44.7 & & 2.8 & 4.93 & 1.87 & -4.2 & 27.0 & 0.12 & $2 \%$ & 0.54 \\
\hline \multirow[t]{2}{*}{$0502+041$} & $X$ & 34.1 & -0.1 & 3.6 & 1.33 & 0.66 & -7.6 & 22.9 & 0.08 & $1 \%$ & 0.23 \\
\hline & $S$ & 37.2 & & 2.7 & 4.88 & 2.59 & -3.6 & 28.3 & 0.11 & $1 \%$ & 0.28 \\
\hline \multirow[t]{2}{*}{ 0519-074 } & $X$ & 41.1 & 0.3 & 3.7 & 1.02 & 0.63 & -3.5 & 22.9 & 0.08 & $1 \%$ & 0.23 \\
\hline & $S$ & 27.6 & & 2.8 & 3.85 & 2.21 & -0.3 & 34.6 & 0.13 & $2 \%$ & 0.69 \\
\hline \multirow[t]{2}{*}{$0651+428$} & $X$ & 85.6 & -0.1 & 2.8 & 1.08 & 0.52 & 7.6 & 44.7 & 0.14 & $1 \%$ & 0.45 \\
\hline & $S$ & 98.7 & & 0.9 & 3.96 & 1.98 & 9.5 & 66.8 & 0.14 & $1 \%$ & 0.67 \\
\hline
\end{tabular}

Notes. The total flux densities (integrated from the $X$ - and $S$-band images) are given in mJy. The corresponding $\mathrm{S} / \mathrm{X}$-spectral index is noted $\alpha_{\mathrm{sx}}$, while the continuous structure index is labelled SI. The parameters of the 105 naturally weighted VLBI images at $X$ - and $S$-bands are listed. The restoring beam is an elliptical Gaussian with FWHM (Full Width at Half Maximum) major axis $a$ and minor axis $b$ (units in mas), with major axis in position angle $\phi$ (measured north through east; units in degrees). The columns "Peak" and "rms" represent respectively the peak brightness and the rms (root mean square) of the residuals of the final hybrid image (units in mJy/beam). The first contour level (percentage of the peak and corresponding value in $\mathrm{mJy} / \mathrm{beam}$ ) for each map is also given.

noise is $0.080 \mathrm{mJy} / \mathrm{beam}$ at $X$-band (values ranging from 0.033 to $0.158 \mathrm{mJy} / \mathrm{beam}$ ) and $0.117 \mathrm{mJy} / \mathrm{beam}$ at $S$-band (values ranging from 0.083 to $0.233 \mathrm{mJy} / \mathrm{beam}$ ) except for $1007+716$ which suffers from a lack of observations. This compares well with the theoretical image thermal noise $(\sigma)$ at $X$ - and $S$-bands, which are 0.050 and $0.082 \mathrm{mJy} / \mathrm{beam}$, respectively (or 0.086 and $0.160 \mathrm{mJy} / \mathrm{beam}$, respectively, without the 70-m Robledo telescope).
The total flux densities of the targets, derived from integrating the GC030 images, range from 23 to $222 \mathrm{mJy}$ at $X$-band (median value $61 \mathrm{mJy}$ ) and from 22 to $397 \mathrm{mJy}$ at $S$-band (median value $65 \mathrm{mJy}$ ). These values are listed in Table 3 and their distributions are plotted in Fig. 4. These flux densities were compared to those determined during EC025A, as a check of the calibration in this experiment. At $X$-band they showed consistency within $10 \%$ (mean flux density ratio of $1.1 \pm 0.3$ ), with 


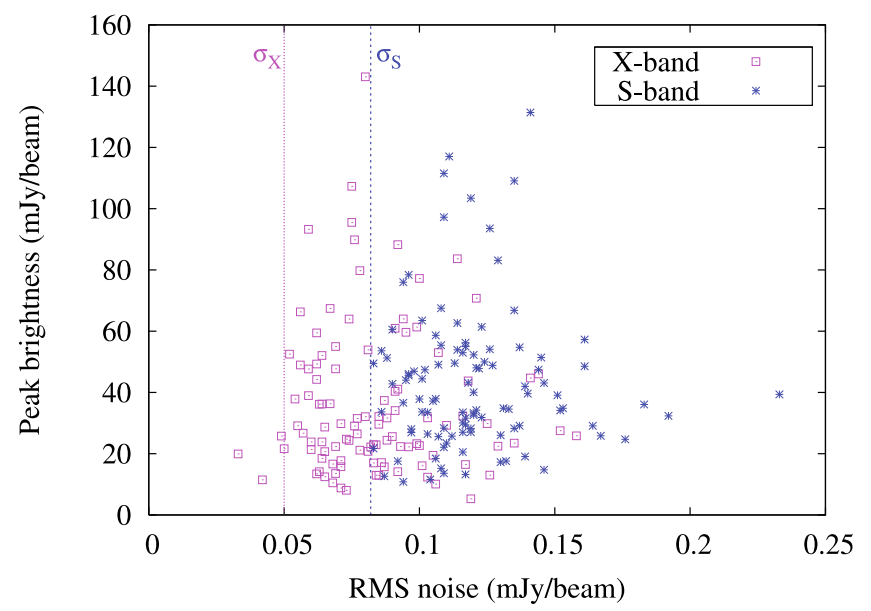

Fig. 3. Peak brightness versus rms noise for the 105 VLBI targets imaged during GC030. The theoretical image thermal noise at $S$ - and $X$-bands is also shown $\left(\sigma_{S}=0.082\right.$ and $\left.\sigma_{X}=0.050 \mathrm{mJy} / \mathrm{beam}\right)$.
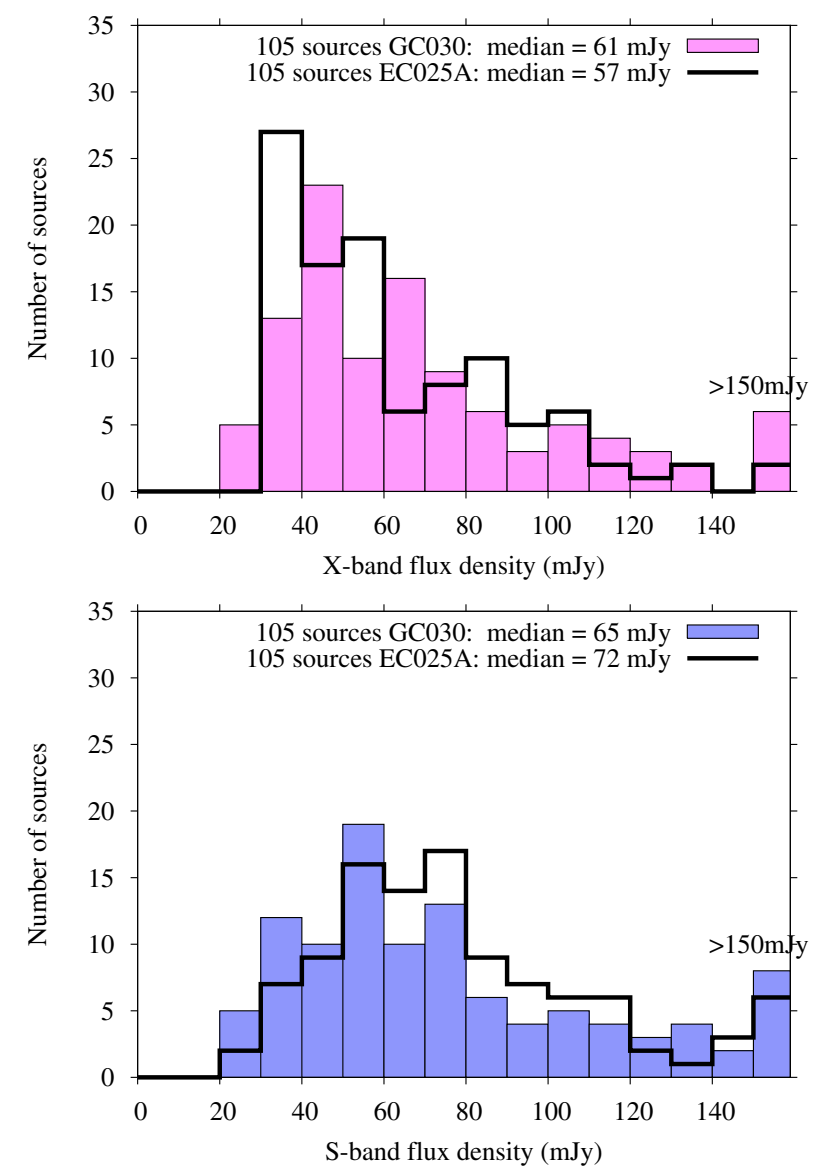

Fig. 4. Distribution of the total flux densities at $X$ - and $S$-bands for the 105 sources observed during GC030. The corresponding mean correlated flux density distribution determined during EC025A for the same sources is plotted in black.

the GC030 flux densities greater than those from EC025A. At $S$ band the comparison also showed consistency within $10 \%$ (mean flux density ratio of $0.9 \pm 0.1$ ) but with the GC030 flux densities less than those from EC025A. Overall, this indicates that the source strengths derived from EC025 can be relied upon for planning the imaging of the remaining sources. A comparison

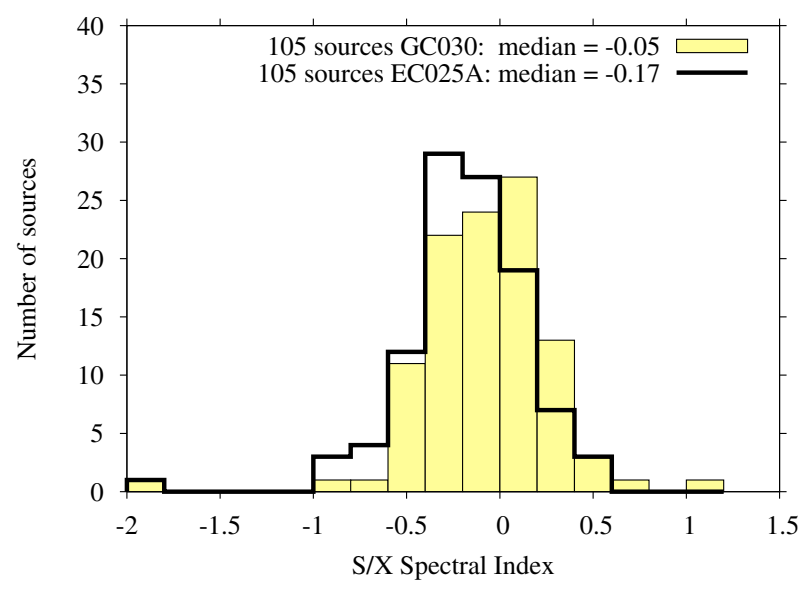

Fig. 5. Distribution of the spectral index $\alpha_{\mathrm{sx}}$ for the 105 sources observed during GC030. The S/X spectral index distribution determined during EC025A for the same sources is plotted in black.
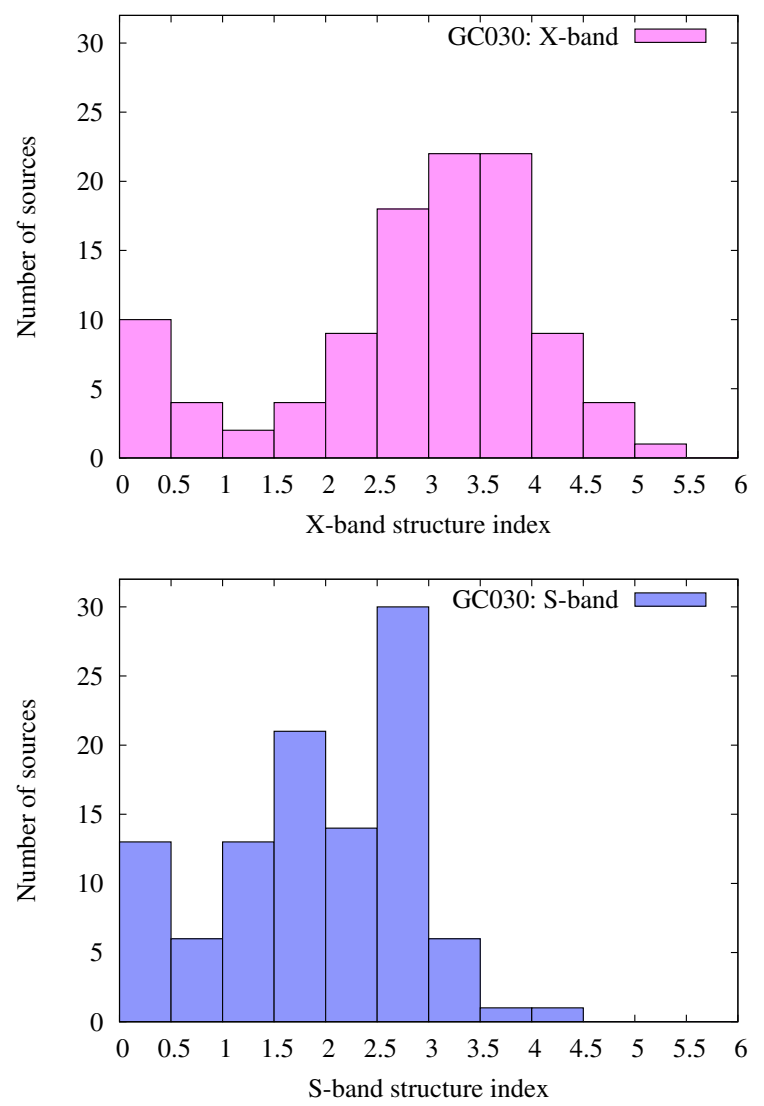

Fig. 6. Distribution of the continuous structure index at $X$-band (upper panel) and $S$-band (lower panel) for the 105 sources observed during GC030.

was also made with six sources in common with VCS6 catalog (Petrov et al. 2008) and it was found that these agree within about $20 \%$, which is satisfactory considering that VCS6 data were taken at least 14 months before.

The spectral index $\alpha_{\mathrm{sx}}$ (defined as $S \propto v^{\alpha_{\mathrm{sx}}}$, where $S$ is the source flux density and $v$ is the frequency) has also been determined (Table 3). In this definition, sources with a compact core are expected to have $\alpha_{\mathrm{sx}}>-0.5$. Figure 5 shows the distribution of $\alpha_{\mathrm{Sx}}$ for the 105 sources imaged during GC030. The 
G. Bourda et al.: VLBI observations of optically-bright extragalactic radio sources. II.
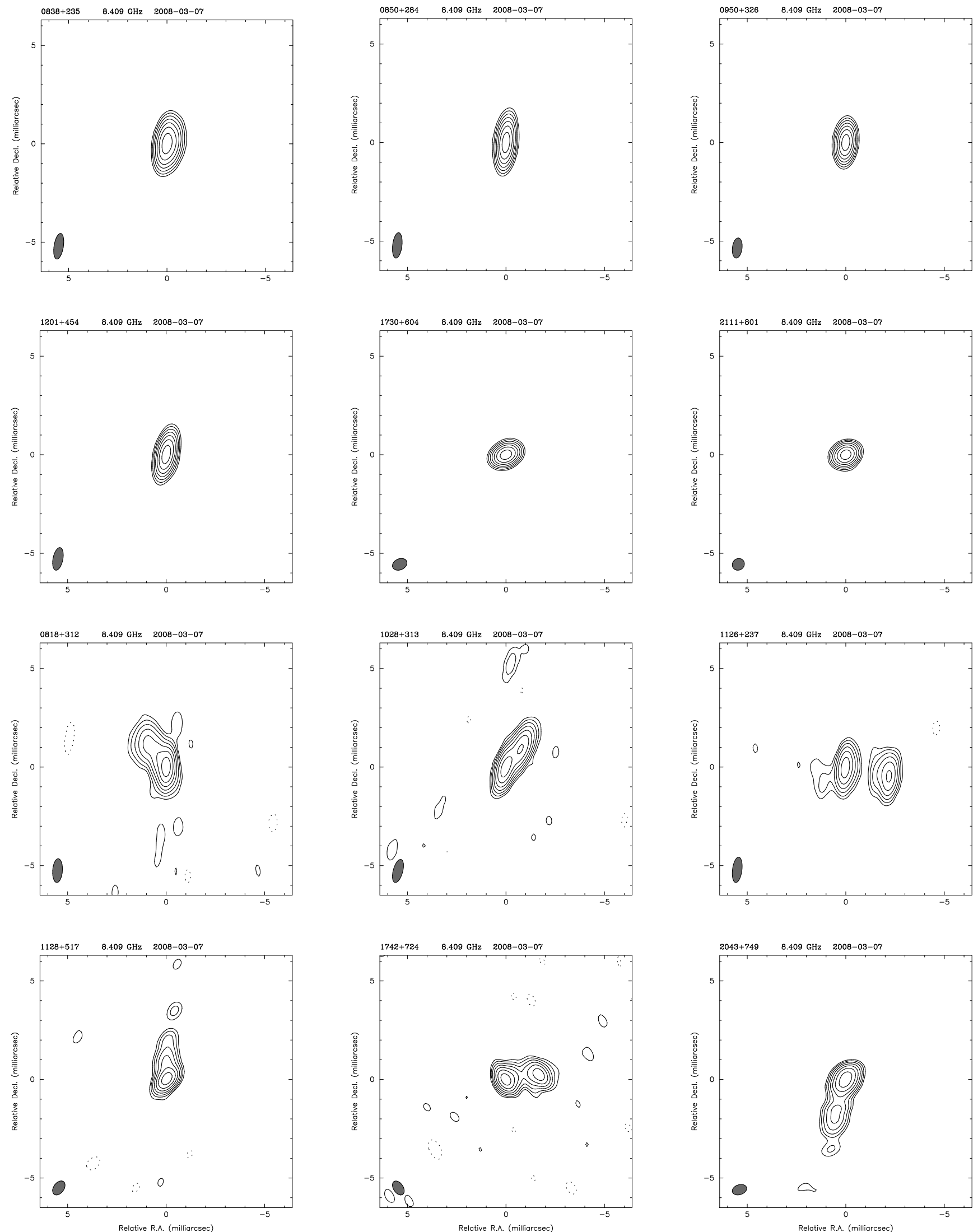

Fig. 7. Examples of $X$-band VLBI maps for sources observed during GC030. Upper panel: six sources suitable for the alignment with Gaia (pointlike sources): $0838+235,0850+284,0950+326,1201+454,1730+604$ and $2111+801$. Lower panel: six sources not suitable for this alignment (sources with extended VLBI structures): $0818+312,1028+313,1126+237,1128+517,1742+724$ and 2043+749. The first contour level is typically $1 \%$ of the peak brightness, with successive contours increasing by a factor of 2 . Image parameters are listed in Table 3. The FWHM Gaussian restoring beam applied to the images is shown as an ellipse in the lower left of each panel. 
Table 4. List of the 47 sources observed during GC030 that were found to be suitable for the alignment with the future Gaia frame (i.e. sources with an $X$-band structure index $<3.0)$.

\begin{tabular}{llllll}
\hline \hline $0049+003$ & $0850+284$ & $1201+454$ & $1556+335$ & $1759+756$ & $2316+238$ \\
$0109+200$ & $0854+334$ & $1212+467$ & $1603+699$ & $1810+522$ & $2322+396$ \\
$0210+515$ & $0907+336$ & $1228+077$ & $1607+604$ & $1811+317$ & \\
$0446+074$ & $0950+326$ & $1242+574$ & $1612+378$ & $1818+551$ & \\
$0651+428$ & $0952+338$ & $1312+240$ & $1618+530$ & $1838+575$ & \\
$0652+426$ & $1020+292$ & $1345+735$ & $1722+119$ & $2052+239$ & \\
$0741+294$ & $1032+354$ & $1429+249$ & $1730+604$ & $2111+801$ & \\
$0838+235$ & $1034+574$ & $1518+162$ & $1741+597$ & $2128+333$ & \\
$0838+456$ & $1145+321$ & $1535+231$ & $1753+338$ & $2247+381$ & \\
\hline
\end{tabular}

median value is -0.05 and 97 of the 105 sources $(\sim 92 \%)$ have $\alpha_{\mathrm{Sx}}>-0.5$. The distribution of the $\mathrm{S} / \mathrm{X}$ spectral index determined during EC025A is also plotted. The two distributions are in good agreement, as expected from the previous comparison of the flux densities.

\section{Discussion}

On the milliarcsecond scale most extragalactic radio sources exhibit spatially extended structures that vary in both time and frequency. As shown by Charlot (1990), such structures may introduce significant errors in VLBI delay measurements which degrade the source position accuracy. The astrometric suitability of the sources may be estimated from the observed structures through the so-called "structure index", first introduced by Fey \& Charlot (1997) (see also Fey \& Charlot 2000).

The structure index (SI) indicates the expected magnitude of the effects of intrinsic source structure on VLBI delay observations, according to the median value of the structure delay corrections ( $\left.\tau_{\text {median }}\right)$ calculated for all projected VLBI baselines that might be observed, using the algorithm devised by Charlot (1990). While Fey \& Charlot (1997) separated sources into four categories, with values of the structure index ranging from 1 to 4 , a continuous scale was adopted for the present work (as also done for the ICRF2; see IERS Technical Note 35). It is defined as follows:

$\mathrm{SI}=1+2 \log \left(\tau_{\text {median }}\right)$

where $\tau_{\text {median }}$ is expressed in picoseconds (ps). Additionally, SI values are constrained to be always positive by setting $\mathrm{SI}=0$ when $\log \left(\tau_{\text {median }}\right)<-0.5$ (i.e. $\tau_{\text {median }} \lesssim 0.3 \mathrm{ps}$ ). There is close correspondence at the (discrete) SI boundaries between the continuous SI values defined here and the values defined in Fey \& Charlot (1997) (SI $=1.95$ vs. 2 for $\tau_{\text {median }}=3 \mathrm{ps,} \mathrm{SI}=3.00$ vs. 3 for $\tau_{\text {median }}=10 \mathrm{ps}, \mathrm{SI}=3.95$ vs. 4 for $\tau_{\text {median }}=30 \mathrm{ps}$ ). Therefore the recommendation of Fey \& Charlot (1997) that sources with SI values of 3 or 4 should preferably not be used for high-precision VLBI astrometry remains largely valid with this new definition of the structure index. Accordingly, for the alignment of the VLBI and Gaia frames, only sources with $\mathrm{SI}<3.0$ (i.e. those with the highest astrometric quality) should be used, since the link should be determined with the highest accuracy.

Based on this definition, the continuous structure index values at $X$ - and $S$-bands for each source observed during GC030 were determined (Table 3). As devised by Fey \& Charlot (1997), the median structure corrections were scaled by 1.08 at $X$-band and 0.08 at $S$-band before deriving the structure indices. This was applied in order to account for the factors used in the linear combination of the $S$-band and $X$-band observables when calculating the dual-frequency calibrated delay observation. The distribution of the $X$-band and $S$-band SI is plotted in Fig. 6 . As shown in this figure, about half of the sources (47 sources) have SI values $<3.0$ at $X$-band, which indicates point-like or very compact structures (i.e. sources suitable for the Gaia link). The other half, with $\mathrm{SI} \geq 3.0$, show extended structures and are unsuitable. The list of $\overline{47}$ sources suitable for the Gaia link is given in Table 4. A sample of six such sources is shown in Fig. 7, along with six sources which are unsuitable.

\section{Summary and future prospects}

Based on observations with a global VLBI array, VLBI images at $X$ - and $S$-bands have been obtained for all 105 optically-bright, weak extragalactic radio sources observed, and previously selected as potential candidates to align the radio and Gaia frames. From these, 47 sources have been identified to have high astrometric quality, as required for the alignment. They will be observed during the third step of the project described in Bourda et al. (2010), devoted to determining highly accurate VLBI positions for these sources.

Surveying the rest of the sources detected in our initial experiments (293 sources) is the subject of on-going global observations. Once the full sample has been imaged it will be possible to undertake final comparisons with the ICRF2 catalog (e.g. overall distribution of structure index and flux density, VLBI structure dependance with respect to flux level), as well as to fit Gaussian models to the VLBI images.

Acknowledgements. The authors would like to thank the VLBI friends at the EVN and VLBA observing stations. This work has benefited from research funding from the European Community's sixth Framework Programme under RadioNet R113CT 2003 5058187. The EVN is a joint facility of European, Chinese, South African and other radio astronomy institutes funded by their national research councils. The VLBA is part of the National Radio Astronomy Observatory (NRAO), which is operated by Associated Universities, Inc., under cooperative agreement with the National Science Foundation.

\section{Appendix A: VLBI images}

Figures A.1-A.9 and Figs. A.10-A.18 show the $X$-band and $S$ band VLBI maps, respectively, determined for the 105 target sources observed during GC030. Image parameters are listed in Table 3. The FWHM Gaussian restoring beam applied to the images is shown as an ellipse in the lower left of each panel. 
G. Bourda et al.: VLBI observations of optically-bright extragalactic radio sources. II.
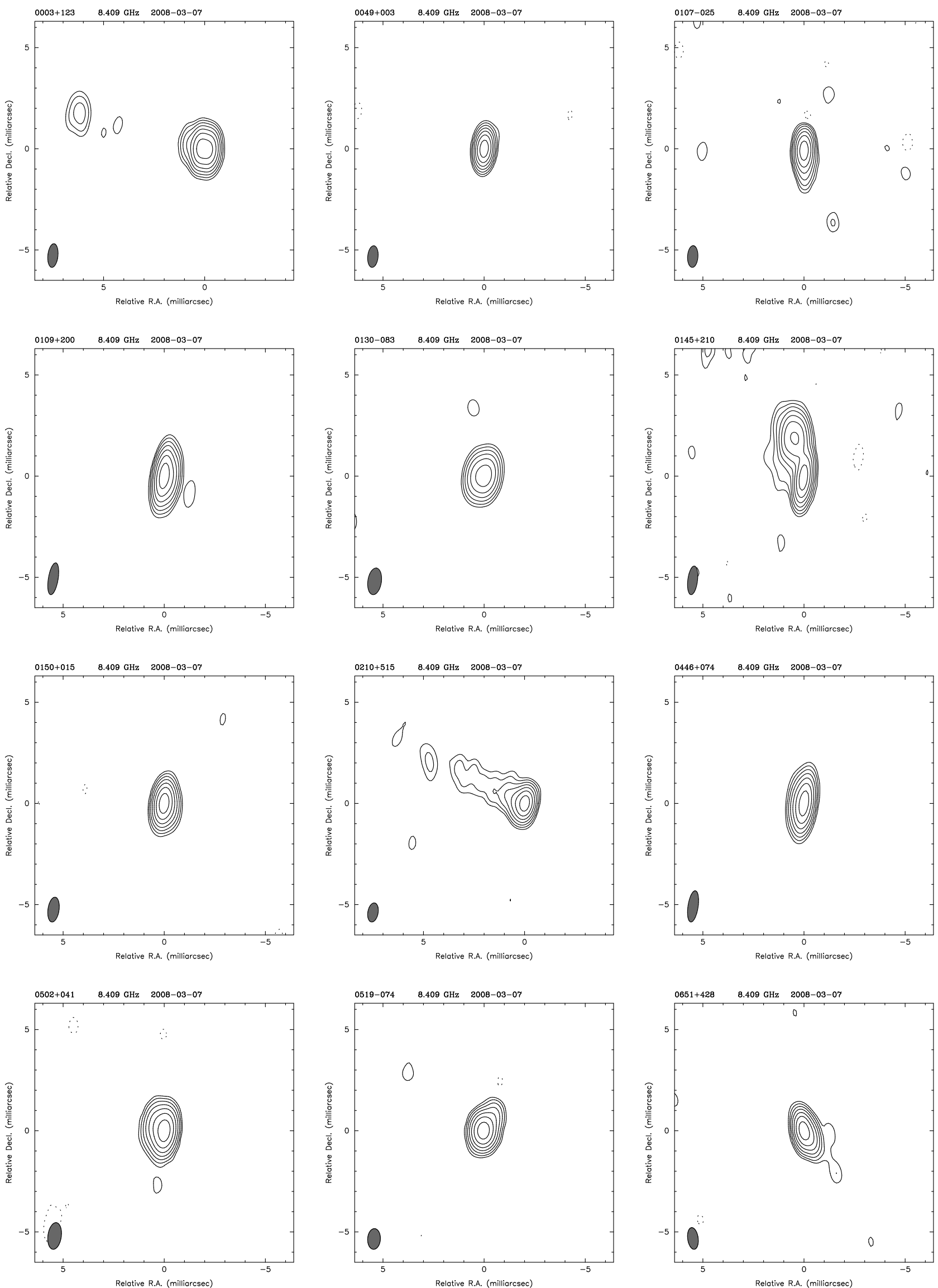

Fig. A.1. $X$-band VLBI maps for the following sources: $0003+123,0049+003,0107-025,0109+200,0130-083,0145+210,0150+015$, $0210+515,0446+074,0502+041,0519-074$ and $0651+428$. 
A\&A 526, A102 (2011)
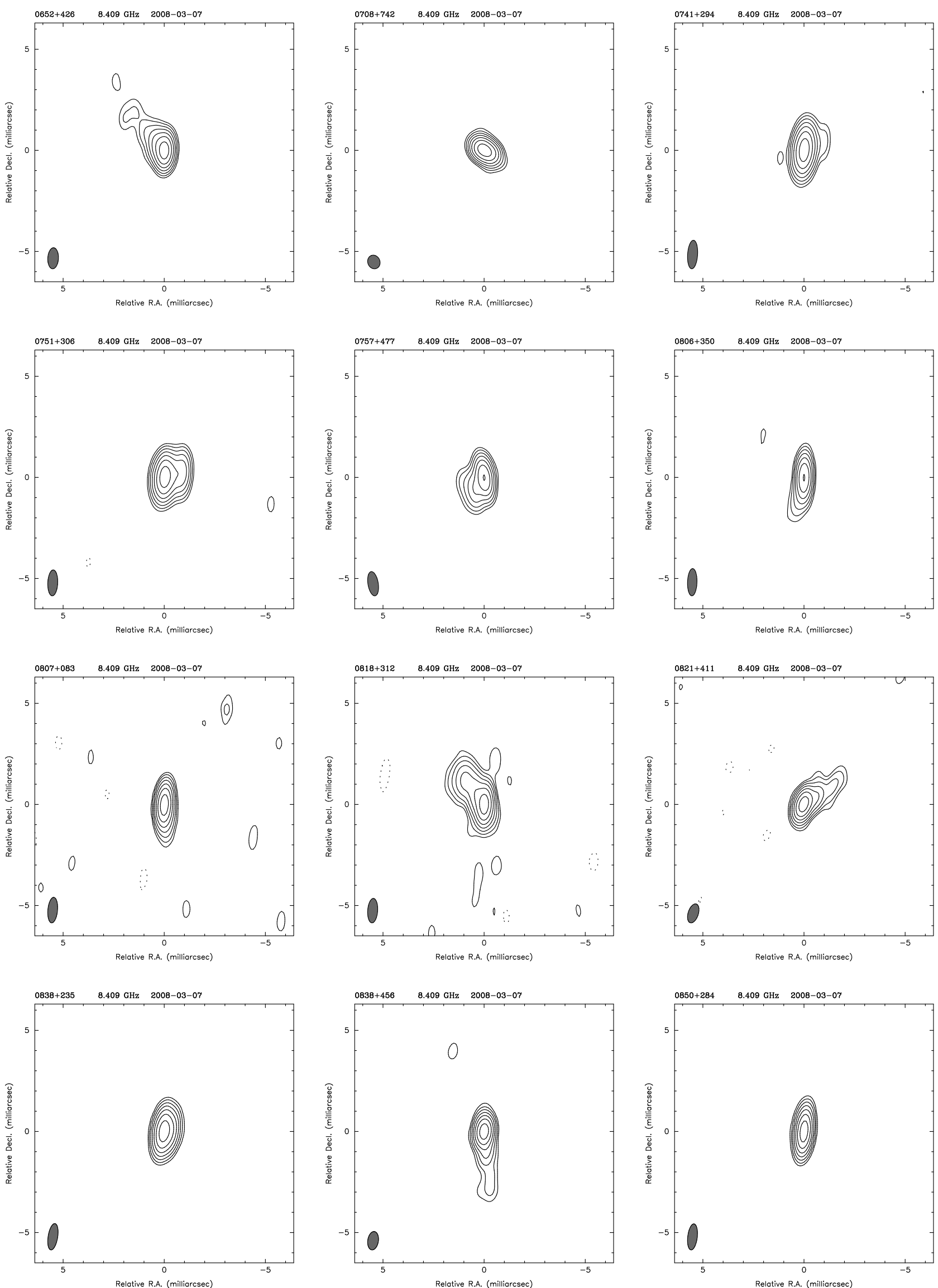

Fig. A.2. $X$-band VLBI maps for the following sources: $0652+426,0708+742,0741+294,0751+306,0757+477,0806+350,0807+083$, $0818+312,0821+411,0838+235,0838+456$ and $0850+284$. 
G. Bourda et al.: VLBI observations of optically-bright extragalactic radio sources. II.
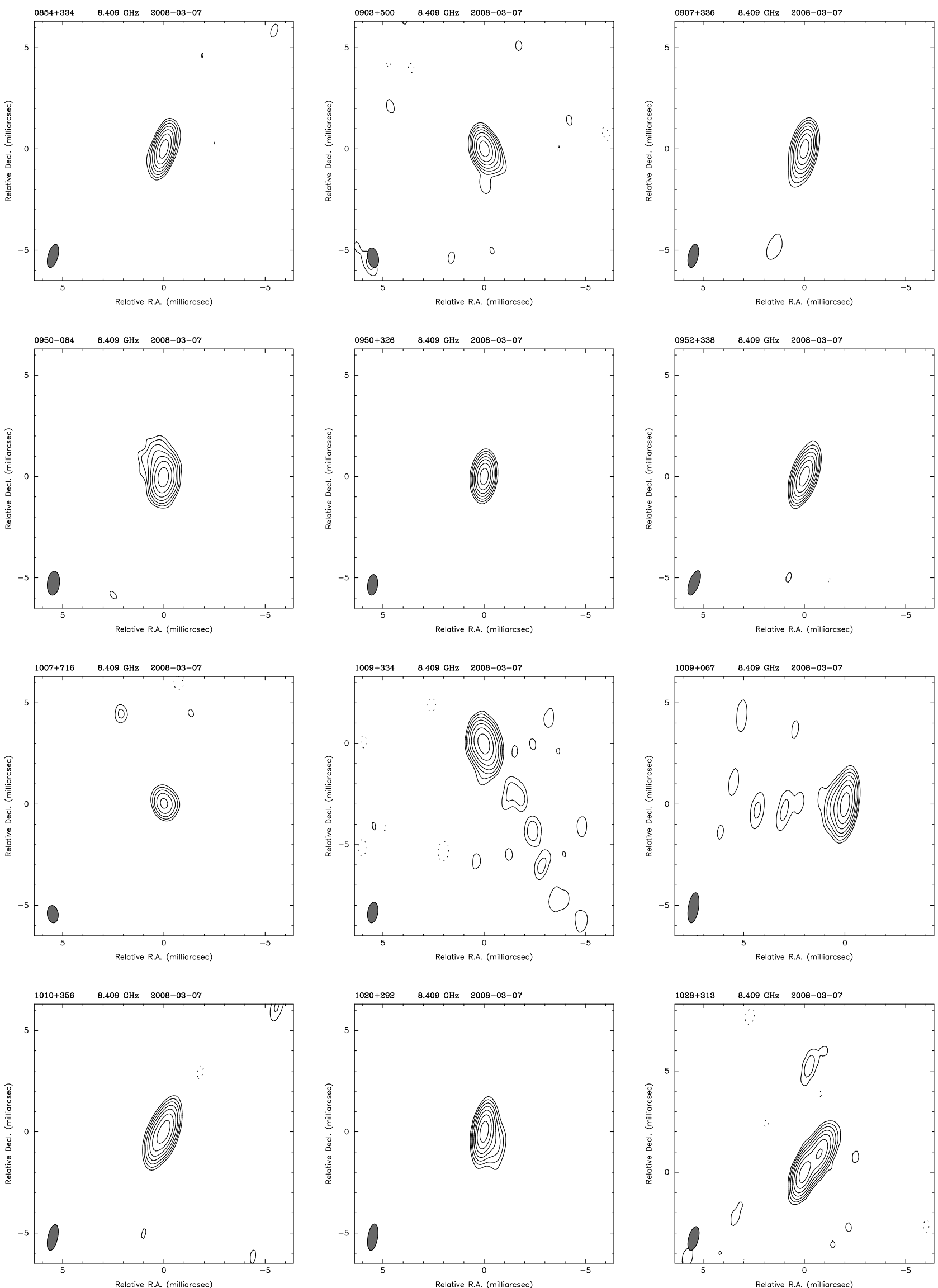

Fig. A.3. $X$-band VLBI maps for the following sources: $0854+334,0903+500,0907+336,0950-084,0950+326,0952+338,1007+716$, $1009+334,1009+067,1010+356,1020+292$ and $1028+313$. 
A\&A 526, A102 (2011)
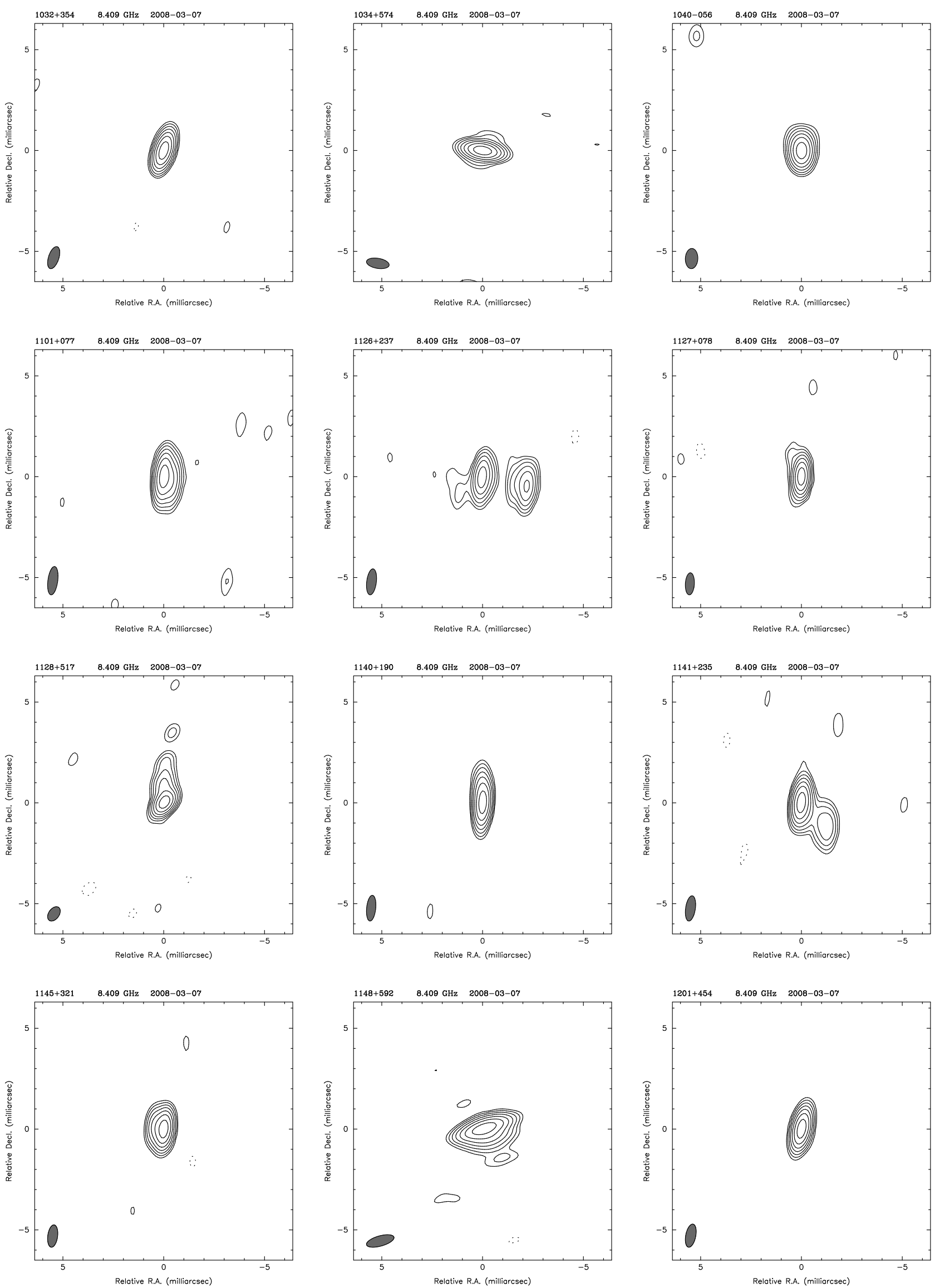

Fig. A.4. $X$-band VLBI maps for the following sources: 1032+354, 1034+574, 1040-056, 1101+077, 1126+237, 1127+078, 1128+517, $1140+190,1141+235,1145+321,1148+592$ and $1201+454$. 
G. Bourda et al.: VLBI observations of optically-bright extragalactic radio sources. II.
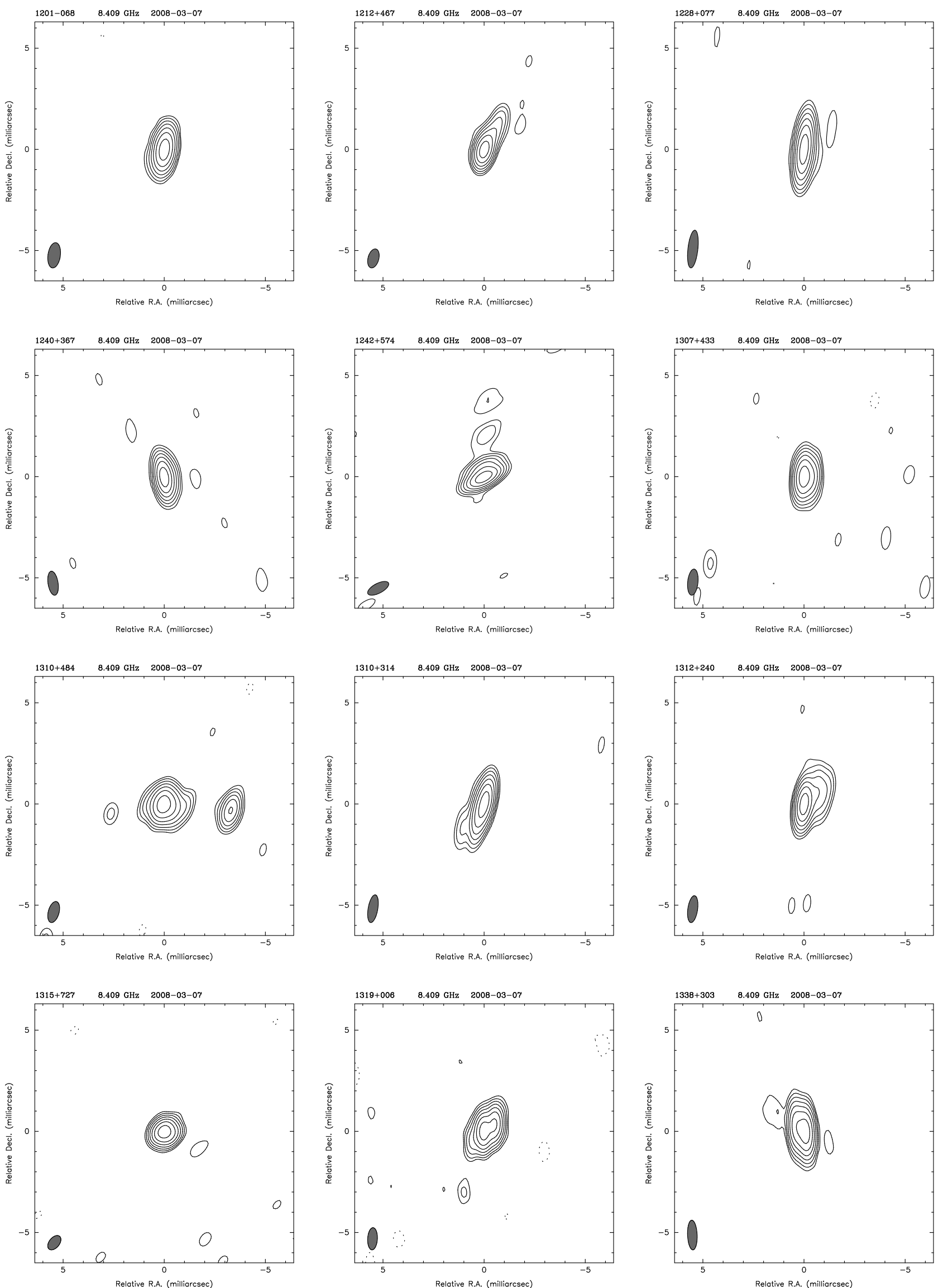

Fig. A.5. $X$-band VLBI maps for the following sources: 1201-068, 1212+467, 1228+077, 1240+367, 1242+574, 1307+433, 1310+484, $1310+314,1312+240,1315+727,1319+006$ and $1338+303$. 
A\&A 526, A102 (2011)
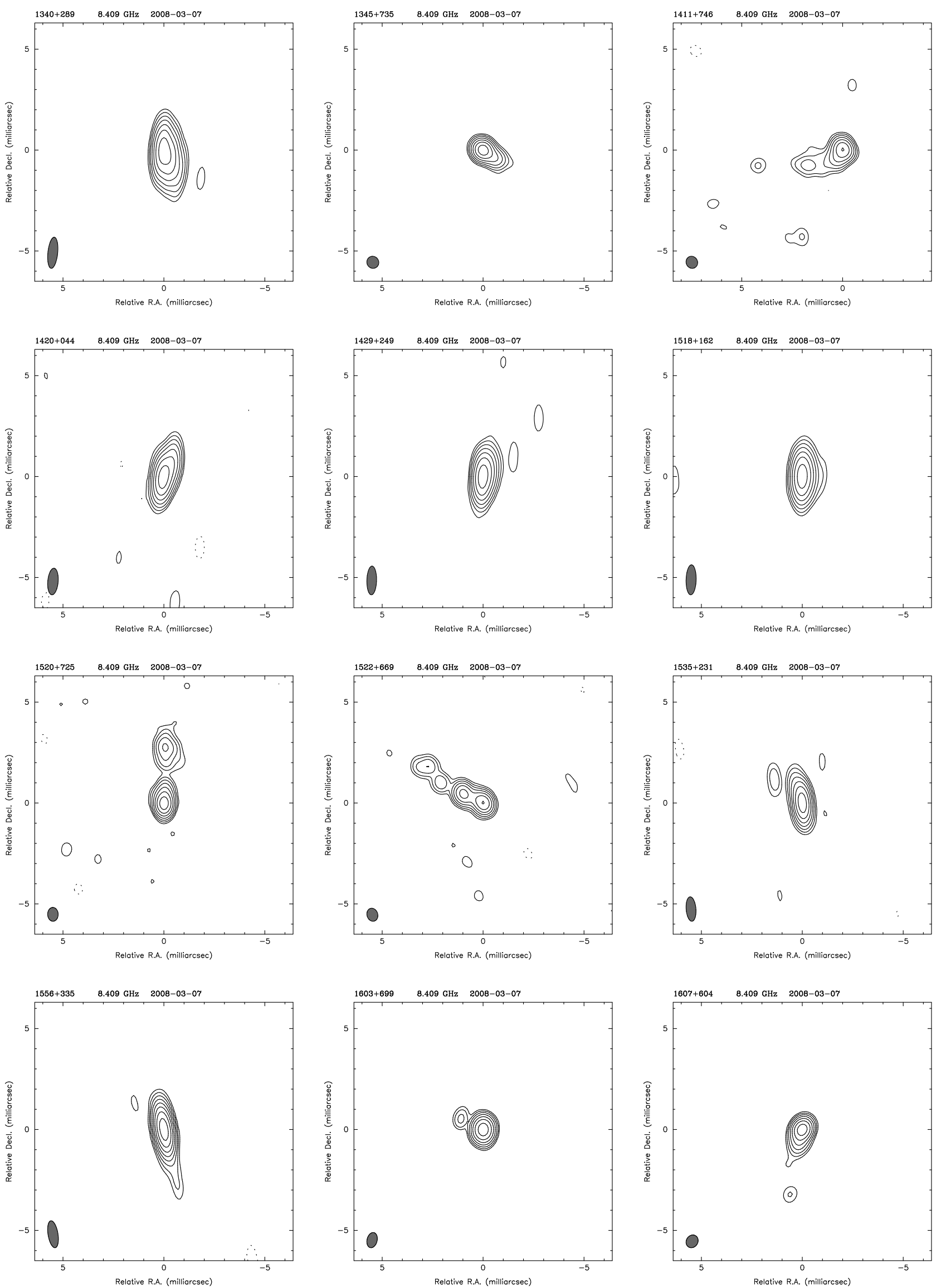

Fig. A.6. $X$-band VLBI maps for the following sources: $1340+289,1345+735,1411+746,1420+044,1429+249,1518+162,1520+725$, $1522+669,1535+231,1556+335,1603+699$ and $1607+604$. 
G. Bourda et al.: VLBI observations of optically-bright extragalactic radio sources. II.
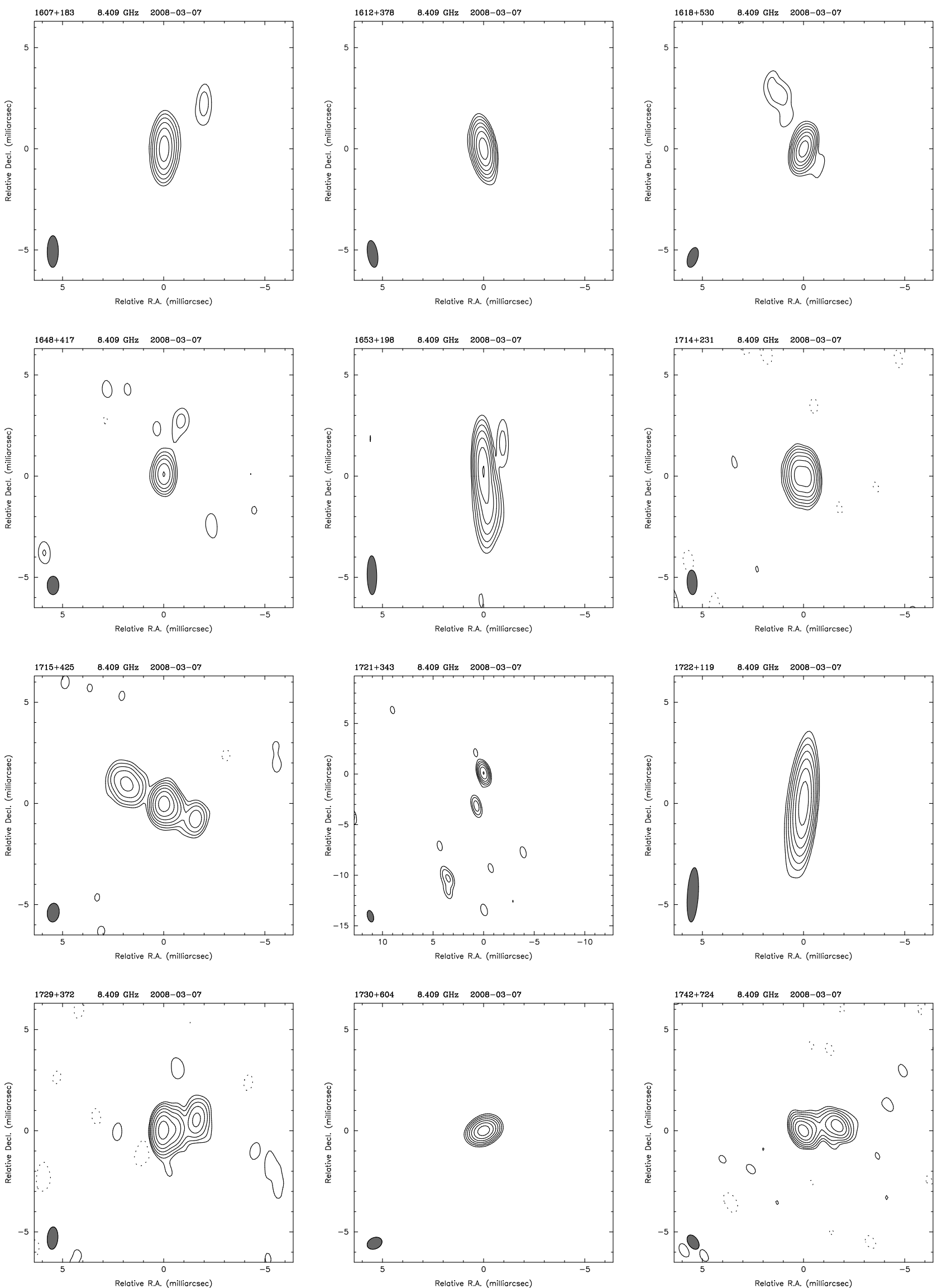

Fig. A.7. $X$-band VLBI maps for the following sources: $1607+183,1612+378,1618+530,1648+417,1653+198,1714+231,1715+425$, $1721+343,1722+119,1729+372,1730+604$ and $1742+724$. 
A\&A 526, A102 (2011)
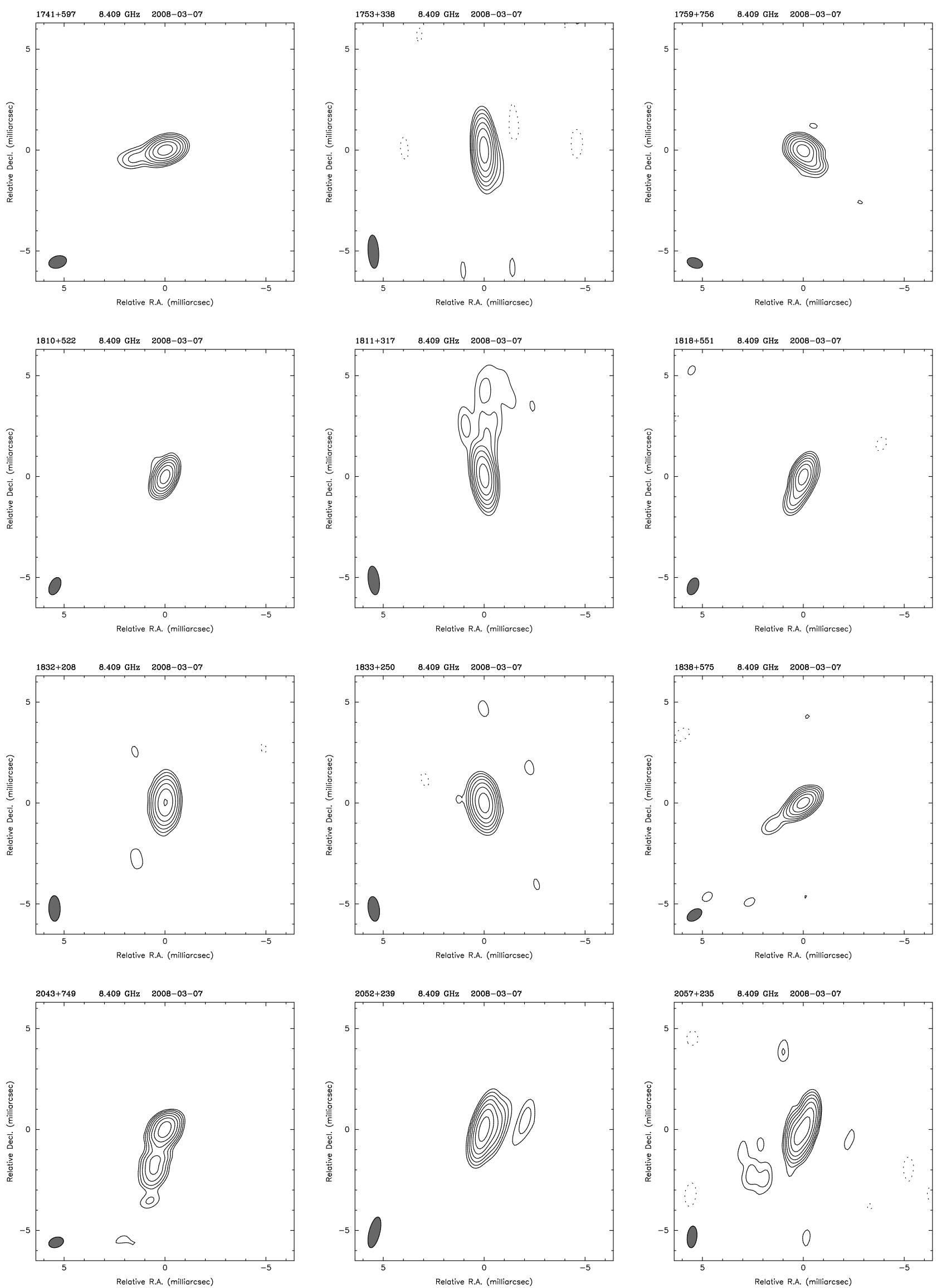

Fig. A.8. $X$-band VLBI maps for the following sources: $1741+597,1753+338,1759+756,1810+522,1811+317,1818+551,1832+208$, $1833+250,1838+575,2043+749,2052+239$ and $2057+235$. 
G. Bourda et al.: VLBI observations of optically-bright extragalactic radio sources. II.
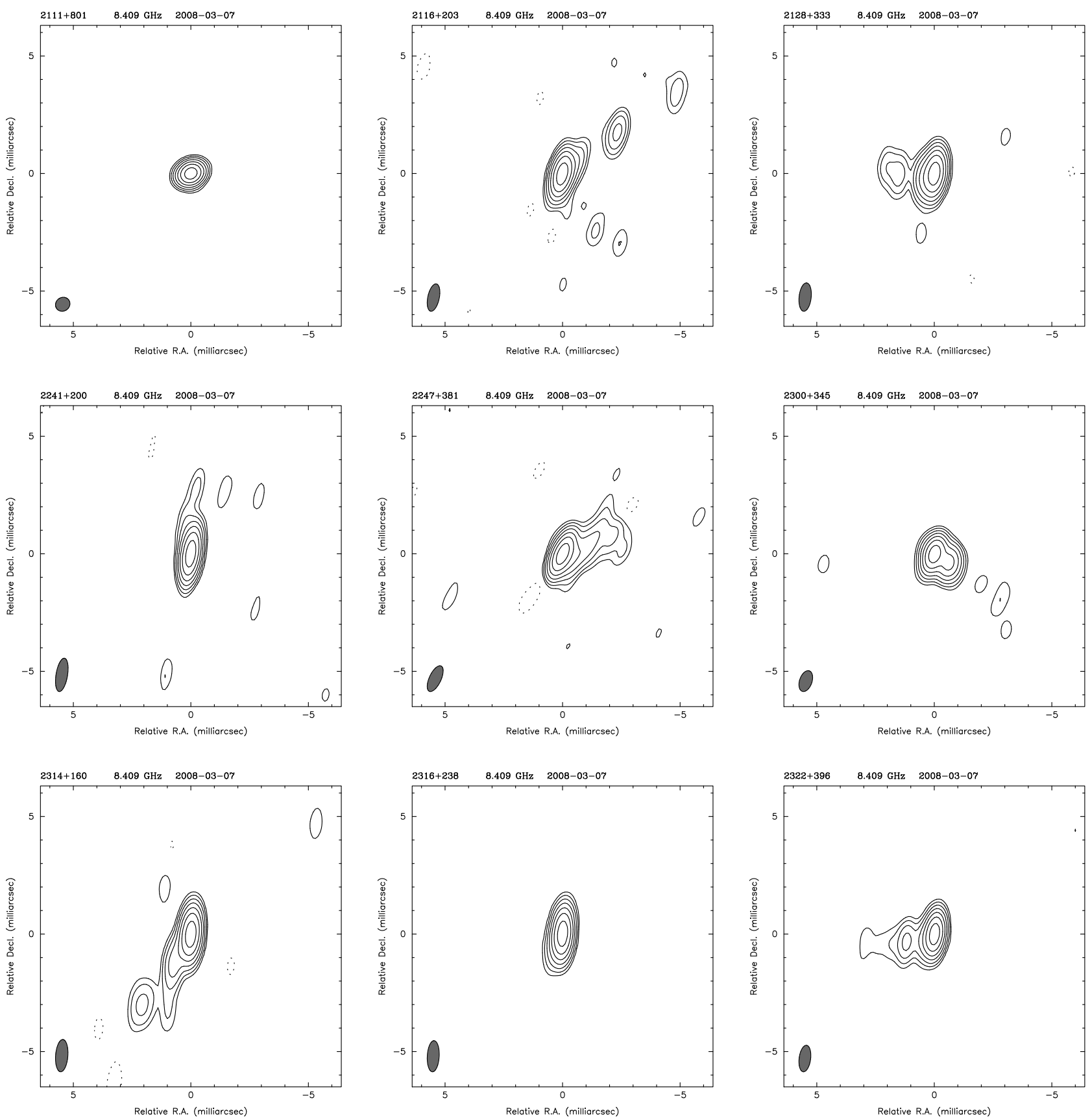

Fig. A.9. $X$-band VLBI maps for the following sources: $2111+801,2116+203,2128+333,2241+200,2247+381,2300+345,2314+160$, $2316+238$ and $2322+396$ 
A\&A 526, A102 (2011)
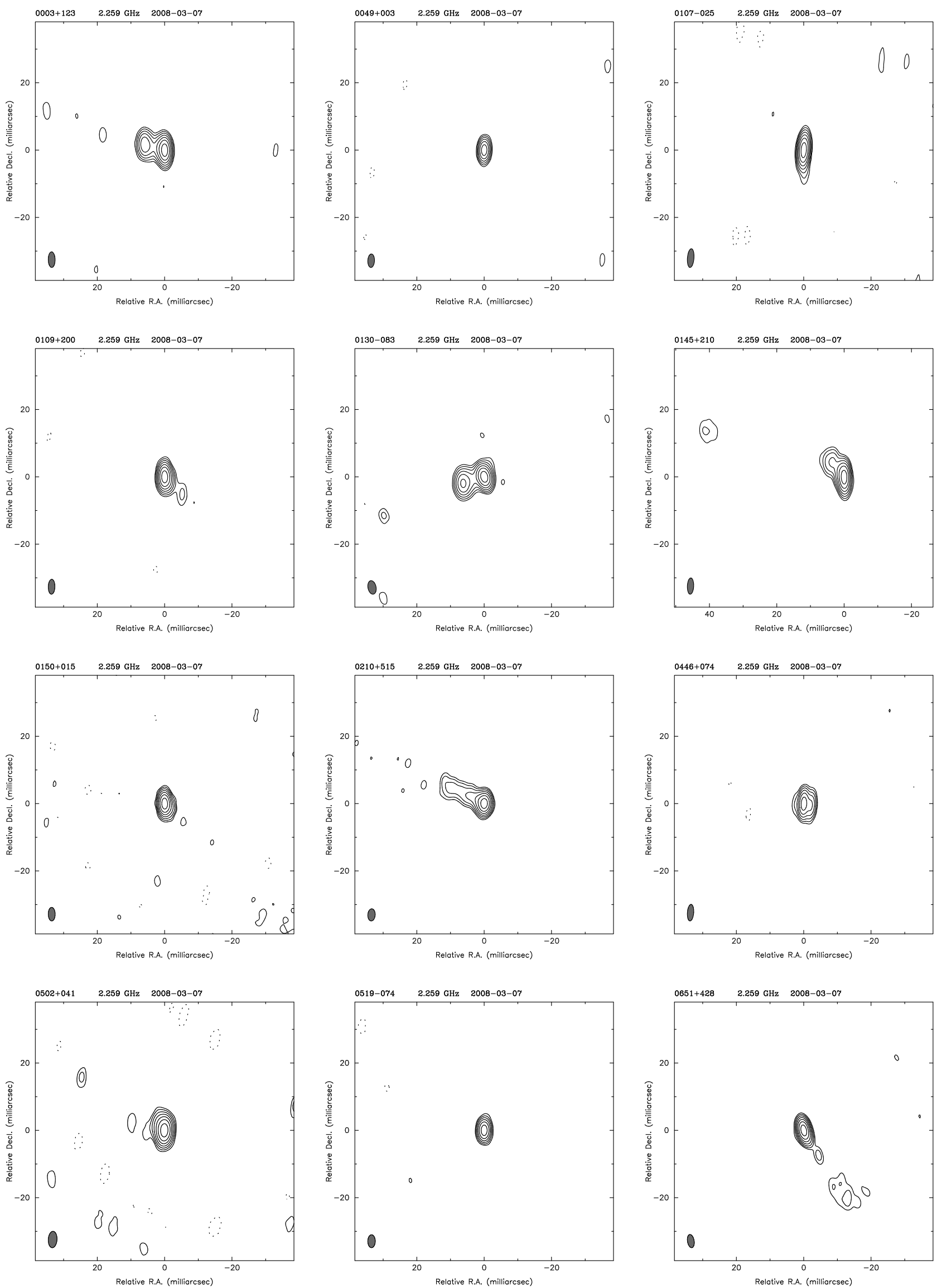

Fig. A.10. S-band VLBI maps for the following sources: 0003+123, 0049+003, 0107-025, 0109+200, 0130-083, 0145+210, 0150+015, $0210+515,0446+074,0502+041,0519-074$ and $0651+428$. 
G. Bourda et al.: VLBI observations of optically-bright extragalactic radio sources. II.
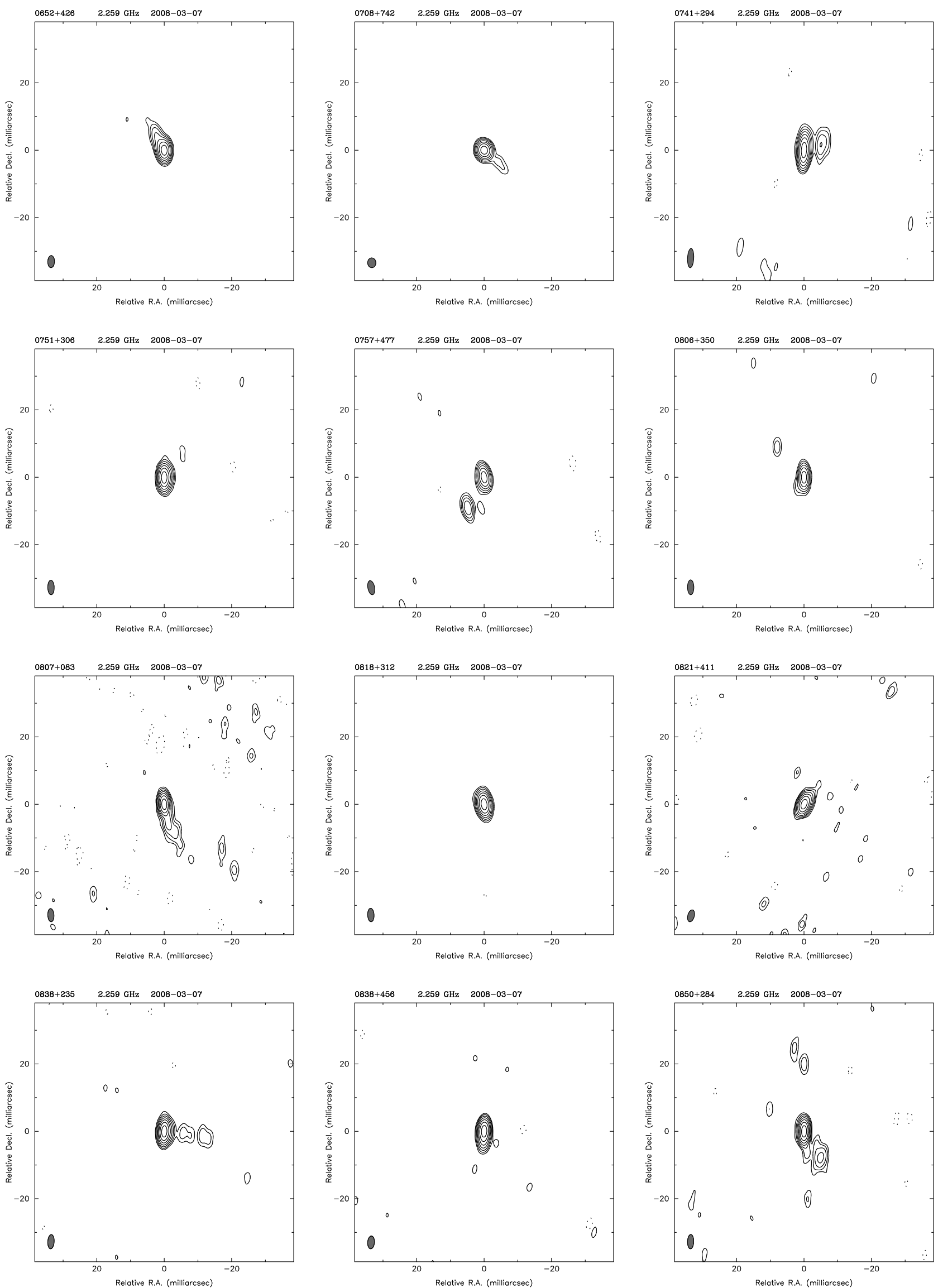

Fig. A.11. S-band VLBI maps for the following sources: $0652+426,0708+742,0741+294,0751+306,0757+477,0806+350,0807+083$, $0818+312,0821+411,0838+235,0838+456$ and $0850+284$. 
A\&A 526, A102 (2011)
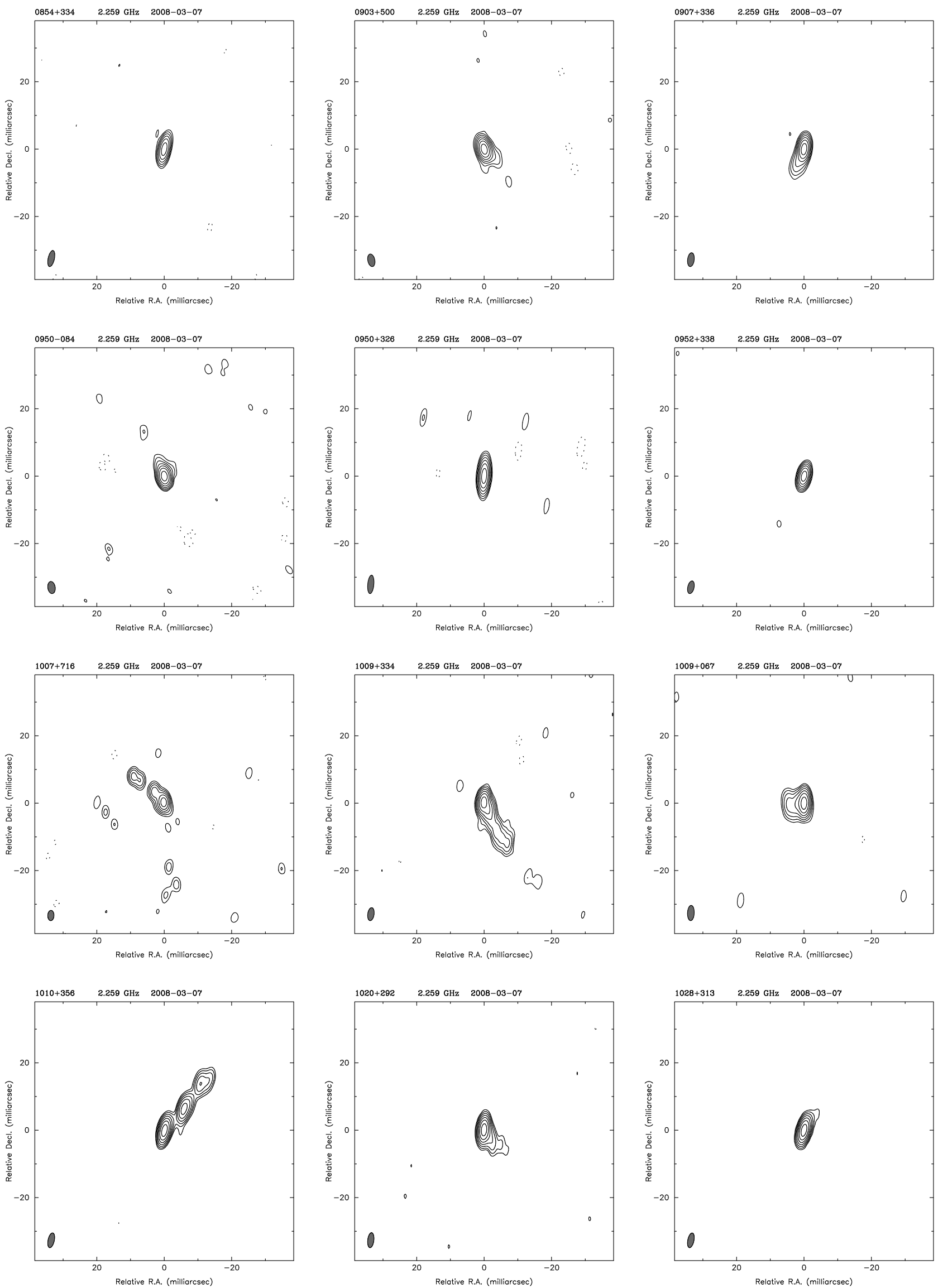

Fig. A.12. $S$-band VLBI maps for the following sources: $0854+334,0903+500,0907+336,0950-084,0950+326,0952+338,1007+716$, $1009+334,1009+067,1010+356,1020+292$ and $1028+313$. 
G. Bourda et al.: VLBI observations of optically-bright extragalactic radio sources. II.
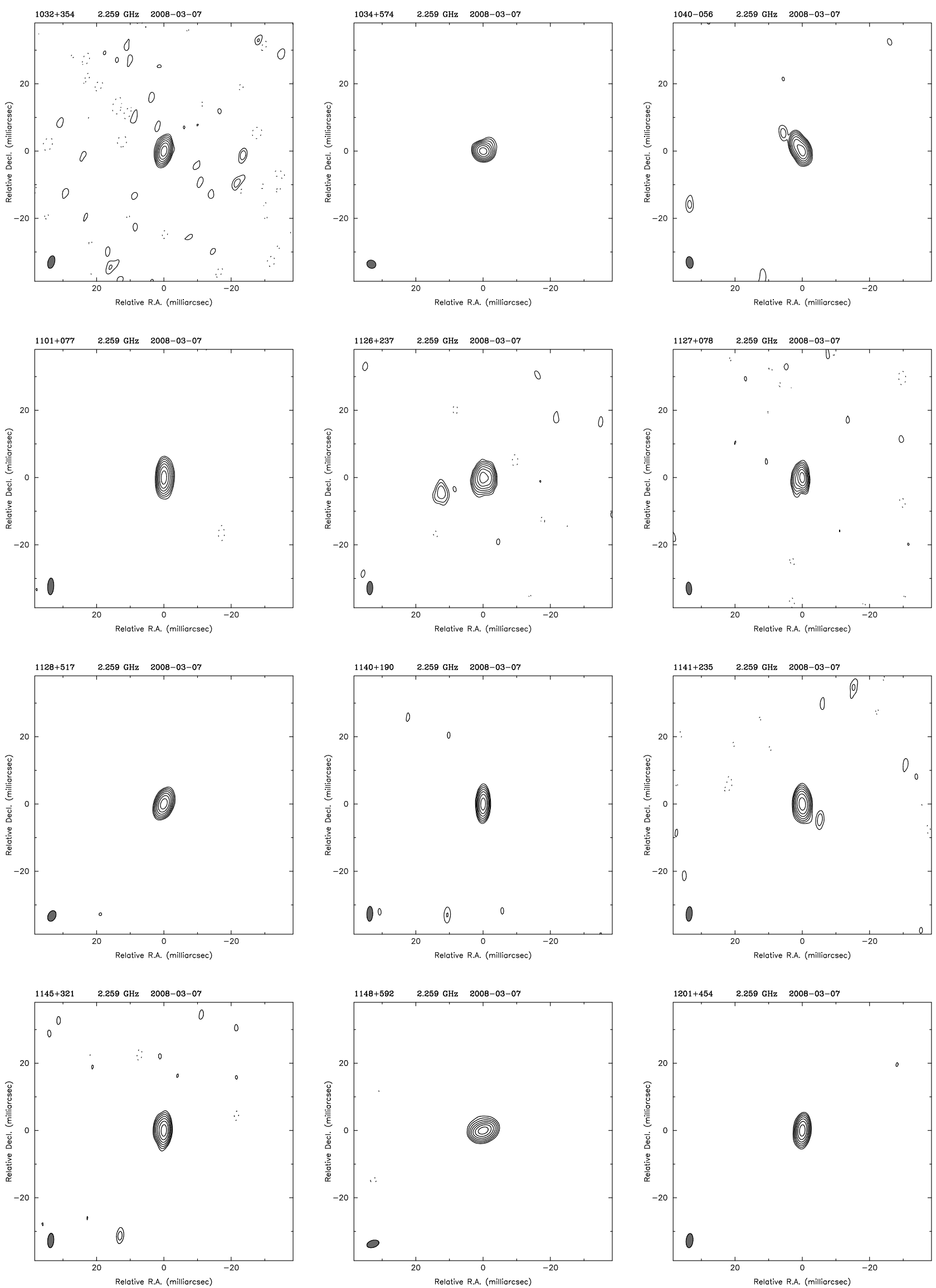

Fig. A.13. S-band VLBI maps for the following sources: 1032+354, 1034+574, 1040-056, 1101+077, 1126+237, 1127+078, 1128+517, $1140+190,1141+235,1145+321,1148+592$ and $1201+454$. 
A\&A 526, A102 (2011)
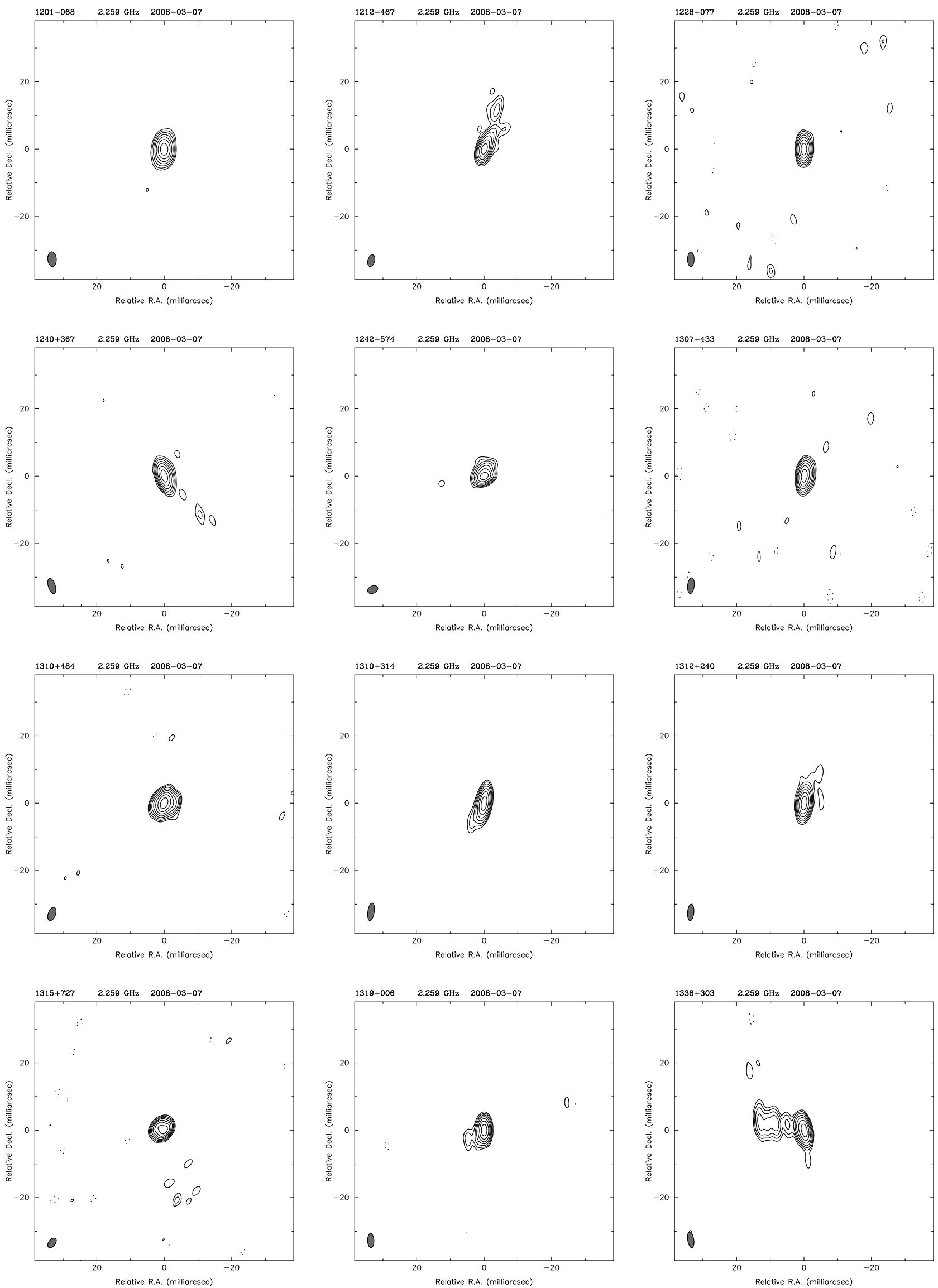

Fig. A.14. S-band VLBI maps for the following sources: 1201-068, 1212+467, 1228+077, 1240+367, 1242+574, 1307+433, 1310+484, $1310+314,1312+240,1315+727,1319+006$ and $1338+303$. 
G. Bourda et al.: VLBI observations of optically-bright extragalactic radio sources. II.
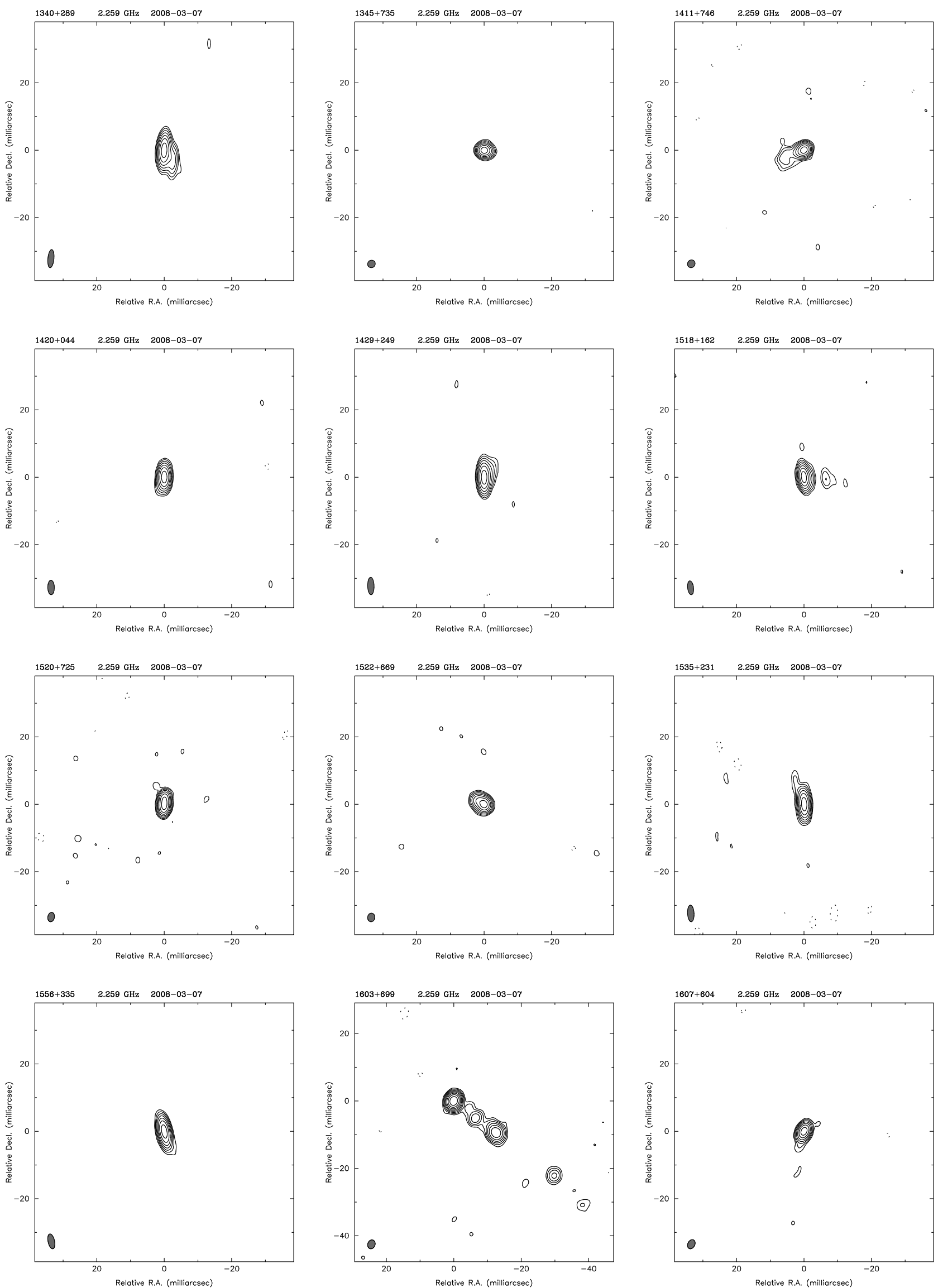

Fig. A.15. $S$-band VLBI maps for the following sources: $1340+289,1345+735,1411+746,1420+044,1429+249,1518+162,1520+725$, $1522+669,1535+231,1556+335,1603+699$ and $1607+604$ 
A\&A 526, A102 (2011)
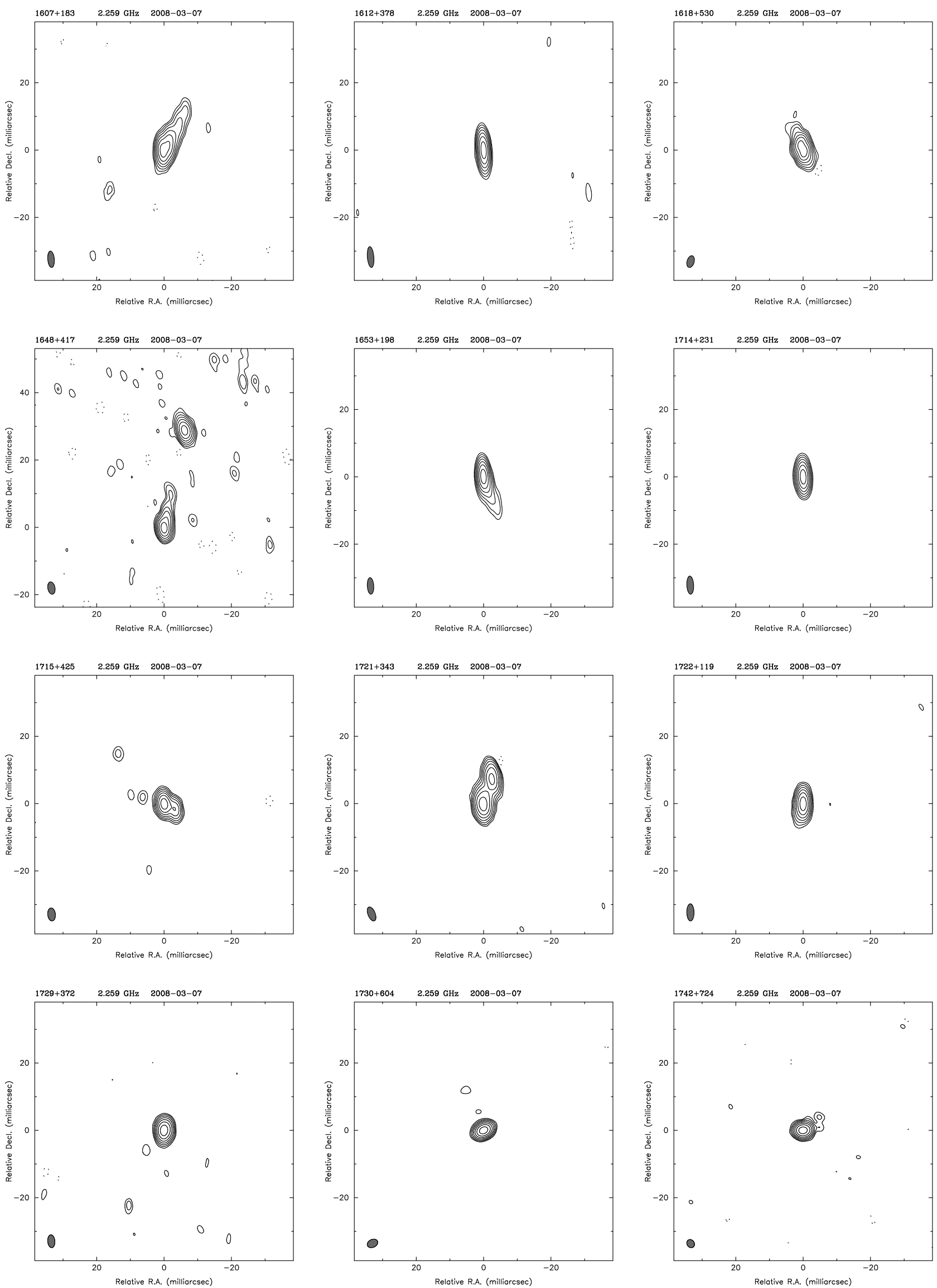

Fig. A.16. $S$-band VLBI maps for the following sources: $1607+183,1612+378,1618+530,1648+417,1653+198,1714+231,1715+425$, $1721+343,1722+119,1729+372,1730+604$ and $1742+724$. 
G. Bourda et al.: VLBI observations of optically-bright extragalactic radio sources. II.
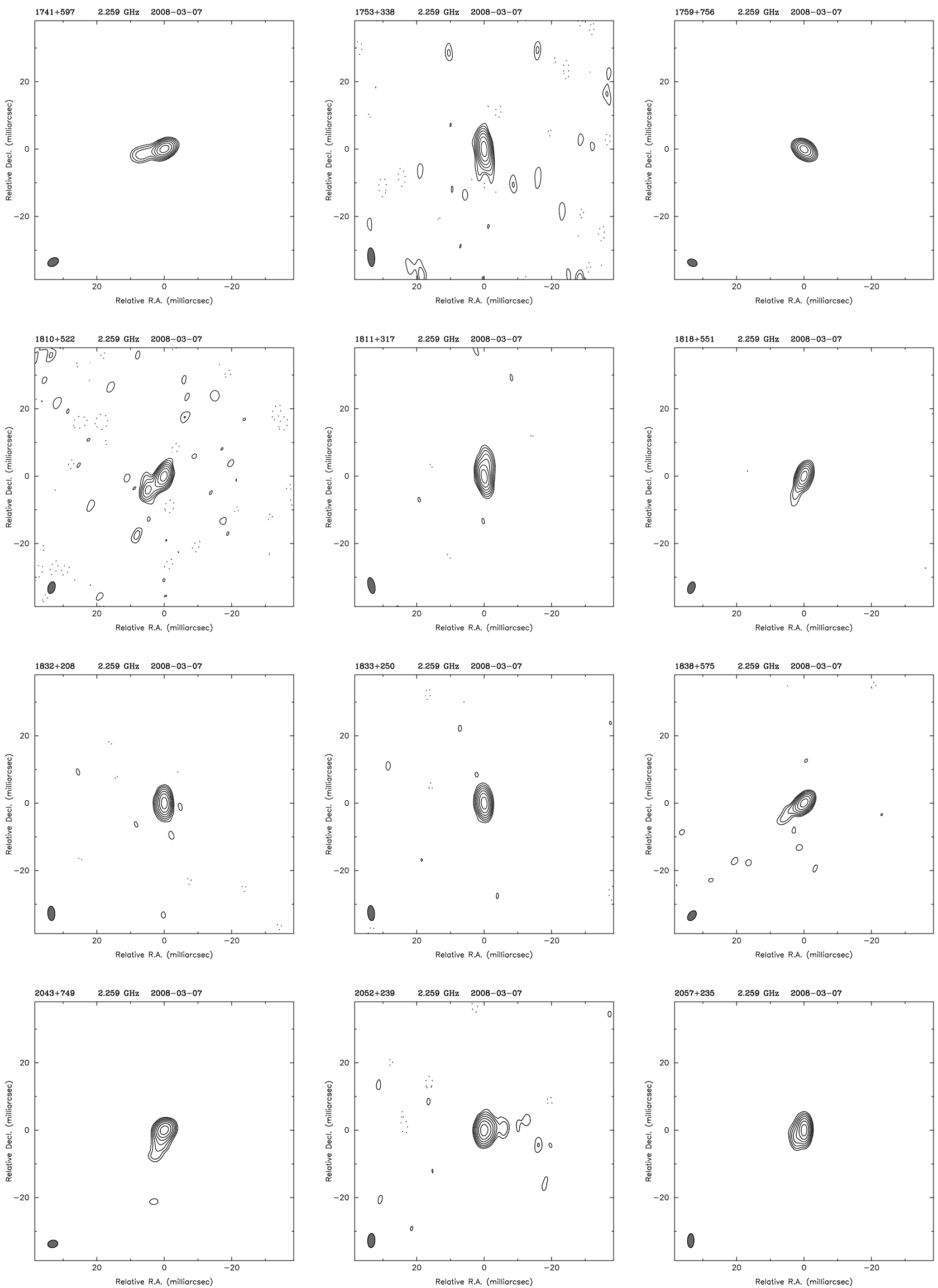

Fig. A.17. $S$-band VLBI maps for the following sources: $1741+597,1753+338,1759+756,1810+522,1811+317,1818+551,1832+208$, $1833+250,1838+575,2043+749,2052+239$ and $2057+235$. 
A\&A 526, A102 (2011)
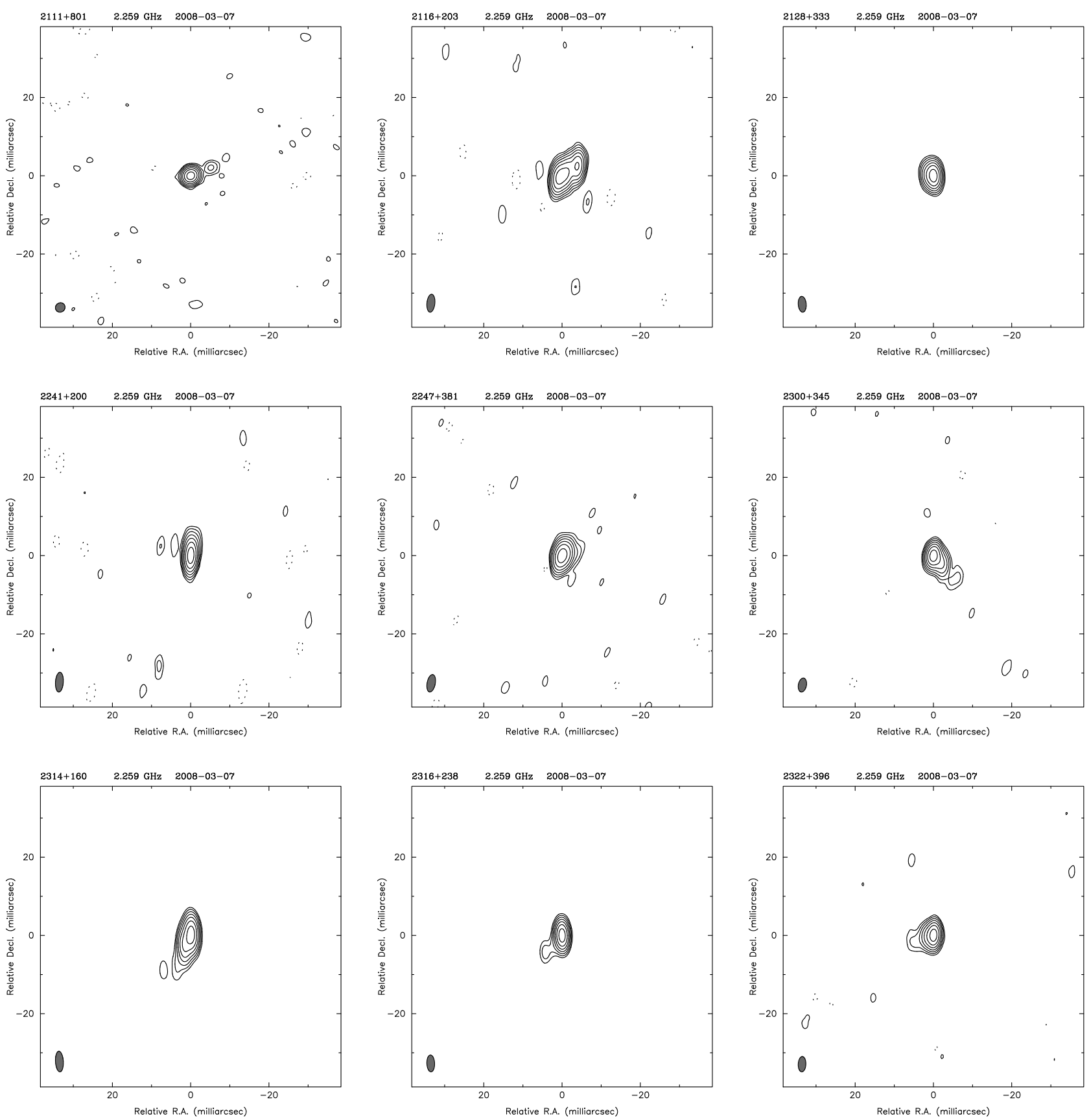

Fig. A.18. $S$-band VLBI maps for the following sources: $2111+801,2116+203,2128+333,2241+200,2247+381,2300+345$, 2314+160, $2316+238$ and $2322+396$ 
G. Bourda et al.: VLBI observations of optically-bright extragalactic radio sources. II.

\section{References}

Bourda, G., Charlot, P., \& Le Campion, J.-F. 2008, A\&A, 490, 403

Bourda, G., Charlot, P., Porcas, R., \& Garrington, S. 2010, A\&A, 520, A113

Charlot, P. 1990, AJ, 99, 1309

Condon, J. J., Cotton, W. D., Greisen, E. W., et al. 1998, AJ, 115, 1693

Fey, A. L., \& Charlot, P. 1997, ApJS, 111, 95

Fey, A. L., \& Charlot, P. 2000, ApJS, 128, 17

Fey, A. L., Ma, C., Arias, E. F., et al. 2004, AJ, 127, 3587

Greisen, E. W. 1988, in Acquisition, Processing and Archiving od Astronomical Images, ed. G. Longo, \& G. Sedmak (Napoli: Observatorio Astronomico di Capodimonte), 125

IERS Technical Note 35 2009, ed. A. L. Fey, D. Gordon, \& C. S. Jacobs (Frankfurt am Main: Verlag des Bundesamts für Kartographie und Geodäsie), The Second Realization of the International Celestial Reference Frame by Very Long Baseline Interferometry
Lindegren, L., Babusiaux, C., Bailer-Jones, C., et al. 2008, in A Giant Step: from Milli- to Micro-arcsecond Astrometry, Proc., ed. W. J. Wenjin, I. Platais, \& M. A. C. Perryman (Cambridge University Press), IAU Symp., 248, 217

Ma, C., Arias, E. F., Eubanks, T. M., et al. 1998, AJ, 116, 516

Napier, P. J., Bagri, D. S., Clark, B. G., et al. 1994, Proc. IEEE, 82, 658

Pearson, T. J., \& Readhead, A. C. S. 1984, ARA\&A, 22, 97

Perryman, M. A. C., de Boer, K. S., Gilmore, G., et al. 2001, A\&A, 369, 339

Petrov, L., Kovalev, Y., Fomalont, E., \& Gordon, D. 2008, AJ, 136, 580

Shepherd, M. C. 1997, in Astronomical Data Analysis Software and Systems VI, ed. G. Hunt, \& H. E. Payne (San Francisco: ASP), ASP Conf. Ser., 125, 77 